\title{
OPERATIONS ON RIGID FORMATIONS OF AUTONOMOUS AGENTS*
}

\author{
TOLGA EREN ${ }^{\dagger}$, BRIAN D. O. ANDERSON ${ }^{\ddagger}$, A. STEPHEN MORSE ${ }^{\S}$, \\ WALTER WHITELEY ${ }^{\top}$, AND PETER N. BELHUMEUR ${ }^{\|}$
}

Dedicated to Sanjoy Mitter on the occasion of his 70th birthday.

\begin{abstract}
This paper is concerned with the maintenance of rigid formations of mobile autonomous agents. A key element in all future multi-agent systems will be the role of sensor and communication networks as an integral part of coordination. Network topologies are critically important for autonomous systems involving mobile underwater, ground and air vehicles and for sensor networks. This paper focuses on developing techniques and strategies for the analysis and design of sensor and network topologies required to achieve a rigid formation for cooperative tasks. Energy efficiency and communication bandwidth are critically important in formations of mobile autonomous agents, and hence strategies that make efficient use of power and energy are beneficial. Therefore, we develop topologies for providing sensing and communications with the minimum number of links, and propose methods requiring the minimum number of changes in the set of links in dynamic missions and maneuvers, including agent departure from a rigid formation, splitting a rigid formation and merging rigid sub-formations. To do this in a systematic manner, it is necessary to develop a framework for modeling agent formations that characterizes the sensing and communication links needed to maintain the formations. The challenge is that a comprehensive theory of such topologies of formations with sensing and communication limitations is in the earliest stage of development. Central to the development of these techniques and strategies will be the use of tools from rigidity theory, and graph theory.
\end{abstract}

1. Introduction. Recent years have seen significant interest in formations of multiple mobile autonomous agents. This interest arises from the broad potential for applications, including formation flight, advanced transportation systems, distributed sensor networks, flocking and schooling, search-and-rescue operations, competitive games, and military reconnaissance and surveillance. A formation is defined as a group of mobile agents moving in real 2- or 3-dimensional space. This paper addresses "rigid formations." A formation is rigid if the distance between each pair of agents does not change over time, at least under ideal conditions. In the context of this paper, "agents" are considered to be autonomous vehicles or sensors such as autonomous

\footnotetext{
*Accepted for publication on February 4, 2004.

${ }^{\dagger}$ Department of Computer Science, Columbia University, New York, NY 10027, E-mail: eren@cs.columbia.edu

${ }^{\ddagger}$ Research School of Information Science and Engineering, Australian National University and National ICT Australia, E-mail: Brian.Anderson@nicta.com.au

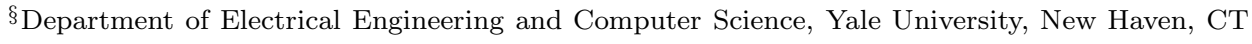
06520, E-mail: as.morse@yale.edu

IDepartment of Mathematics and Statistics, York University, Toronto, Ontario, E-mail: whiteley@mathstat.yorku.ca

"Department of Computer Science, Columbia University, New York, NY 10027, E-mail: belhumeur@cs.columbia.edu
} 
underwater vehicles (AUVs), microsatellites, uninhabited air vehicles (UAVs), mobile ground-based robots, and mobile sensors.

A key element in all future multi-agent systems will be the role of sensor and communications networks as an integral part of coordination. In a rigid formation, distances between agents are held fixed by measurements and information gathered through "sensing and communication links" between agents. One of the challenges in building sensor and communications networks between agents is the "topology" of the network. By topology, we mean the interconnection structure of sensing and communication links among agents. In other words, topology refers to the network's layout. A network's topology determines how different agents in the network are connected to each other. Two networks have the same topology if the interconnection structure is the same, although the networks may differ in physical interconnections, distances between agents, transmission rates, and signal types. Network topologies are critically important for autonomous systems involving mobile underwater, ground and air vehicles, and for sensor networks. Rigid formations with the minimum number of sensing and communication links required to achieve rigidity are called minimally rigid formations.

Formations of autonomous agents usually operate under time-varying conditions where sensor and network topologies need to be restructured. Such conditions can be changes in the environment, obstacles along the trajectories of agents or departures of agents from formation. In this paper, we focus on such topological changes during "operations" on formations. By an operation, we mean missions and maneuvers that include agent departures, splitting, and merging, which result in changes in agent set and/or interconnection structure of sensing and communication links.

First, we consider the problem of splitting rigid formations. By splitting, we mean creating two or more rigid post-split sub-formations from a rigid pre-split formation. When a formation encounters an obstacle, splitting may be useful to maneuver around the obstacle. Instead of all agents moving to the same side of the obstacle, it might be more efficient in terms of trajectory lengths of agents, if some agents move to one side of the obstacle and the others move to the other side.

One strategy to solve the splitting problem would be determining entirely new sets of links for the post-split sub-formations. Olfati-Saber and Murray present such a strategy [20]. When splitting a rigid formation, it is necessary to break the links between agents belonging to different post-split sub-formations. However we can preserve a link between two agents belonging to the same sub-formation. Generating entirely new sets of links for post-split sub-formations is impractical when we can preserve the links that can be maintained. Such a strategy achieves splitting with a minimum number of changes in the topology of sensing and communication links. Our goal is to find such a strategy by inserting the minimum number of links in postsplit non-rigid sub-formations to make each of them rigid while preserving the links 
between agents belonging to the same sub-formations.

To motivate our discussion of splitting a rigid formation, we have the following example:

Example: Consider a rigid formation as shown in Figure 1 in 3-dimensional space. We would like to split the formation in such a way that agents with labels $\{1,2,3,4,5\}$ belong to one post-split sub-formation and agents with labels $\{6,7,8,9,10\}$ belong to the other post-split sub-formation. Splitting can be achieved by removing the links between agents belonging to different post-split sub-formations while preserving the links between agents belonging to the same post-split sub-formations. In this example, the links $(1,6),(3,6),(4,6),(4,7),(4,8),(5,6),(5,7),(5,10)$ (shown with dashed lines) are removed. This results in two non-rigid post-split sub-formations. The splitting problem is to find new sets of links to insert into each non-rigid post-split sub-formation resulting in rigid post-split sub-formations. In this example, those new links are $(3,5)$ and $(6,10)$ shown with double lines.

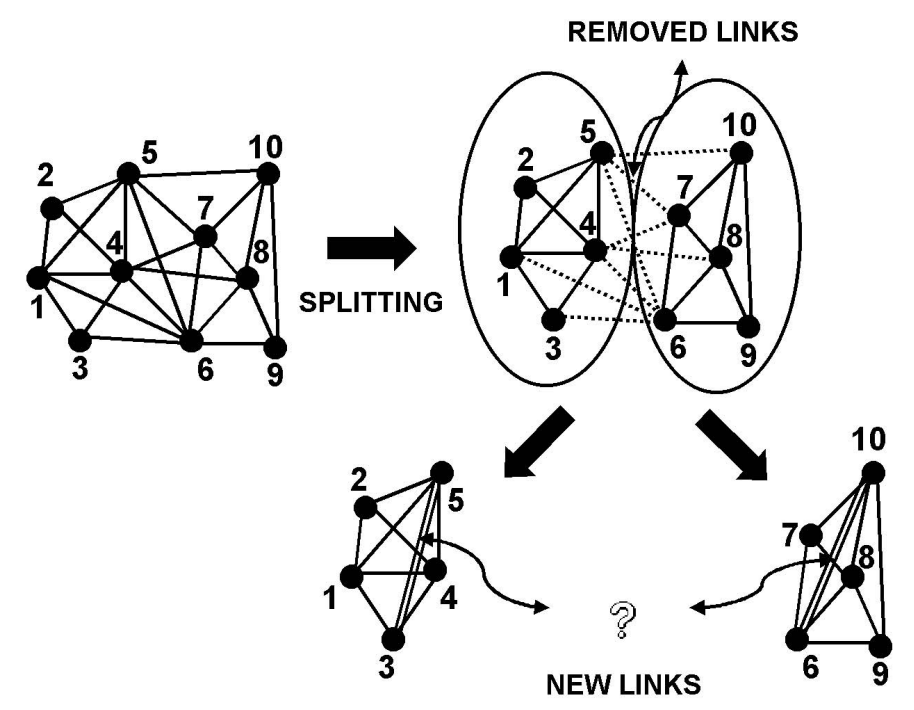

FIG. 1. A rigid formation is split into two sub-formations by removing the links shown with dashed edges, i.e., (1,6), (3,6), (4,6), (4,7), (4,8), (5,6), (5,7), (5,10), which results in two nonrigid sub-formations. The splitting problem is to find the new links that need to be inserted into each non-rigid post-split sub-formation so that each post-split sub-formation regains rigidity. In this example, the new links are (3,5), (6,10) shown with double lines.

Second, we address merging rigid sub-formations. By merging, we mean inserting links between these rigid sub-formations which results in a single post-merged rigid formation. During a merging operation, it is a natural starting point to preserve the links in each pre-merged rigid sub-formation. Hence a reasonable goal is to create a new post-merged rigid formation by inserting a minimum number of links between sub-formations. A merging operation, for example, can be used to create one single 
rigid formation after split sub-formations pass around an obstacle.

As a further application of splitting and merging operations, one can consider using both of these operations together when there is a change in a mission. For example, some changes in sensor and network topologies can be achieved by a series of splitting-merging operations by splitting a formation into two or more sub-formations and then merging these sub-formations into one post-merged formation which has a completely different topology of sensing and communication links.

To motivate our discussion of merging a rigid formation, we have the following example:

Example: Consider two rigid formations in 3-dimensional space as shown in Figure 2. We would like to merge these two formations resulting in a single rigid formation in such a way that all pairs of links in each formation are preserved and a minimum number of links is inserted between these two formations.

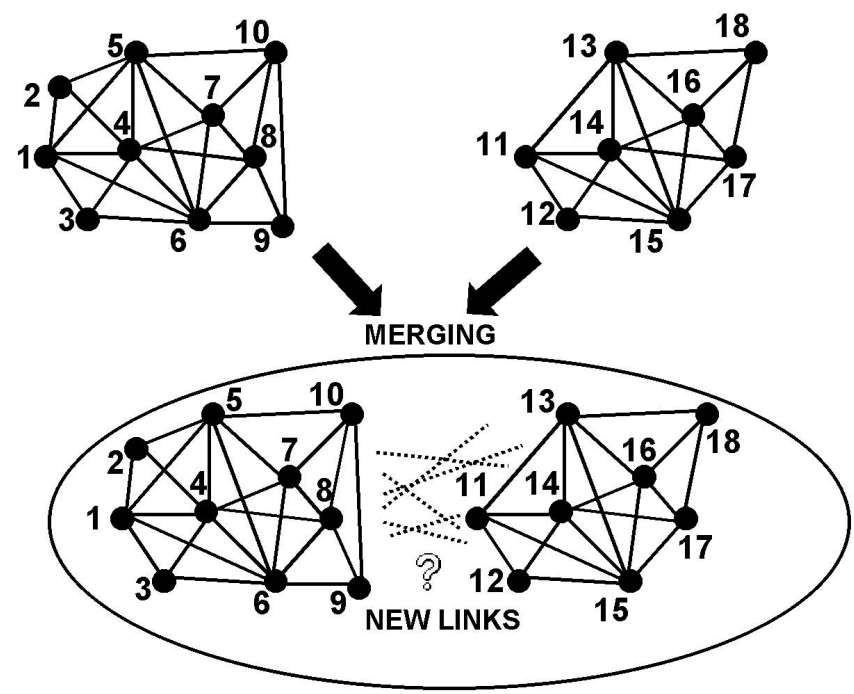

FIG. 2. Two formations are merged to form one single rigid formation. Finding the new links to be inserted between these two formations, which will make the whole formation rigid, is the merging problem.

Merging rigid bodies has been studied in rigidity theory. We refer the reader to Whiteley [27] for a detailed explanation. Here, we use a different approach to find the new links for merging formations. This approach can be used for both splitting and merging formations.

The approach in this paper is based on the strategies developed in Eren et al. [11]. Olfati-Saber and Murray gave an approach to merging sub-formations in 2dimensional space so that the resulting formation is rigid [20]. The approach we develop here allows us to merge two rigid sub-formations with different types of com- 
binations of inserting links including their strategy. Furthermore the approach in this paper also solves the merging problem in 3-dimensional space.

The splitting and merging problems can be considered as special cases of the "minimal cover problem," which we present in the sequel. The minimal cover problem is basically to find new links to insert into a non-rigid formation so that it becomes rigid. To solve the minimal cover problem, we develop a novel procedure. This procedure can be used for creating minimally rigid post-split sub-formations from non-rigid post-split sub-formations and also for creating a minimally rigid post-merged formation from rigid pre-merged sub-formations.

Third, we address the "closing ranks" problem in formations of mobile autonomous agents with limited sensing and communication requirements. By closing ranks, an analogy to the situation in which each unit acts in a coordinated way to meet a challenge, we mean a minimal rearrangement of sensing and communication links between agents in case of agent departures (e.g., an agent is destroyed or has a failure) so that the remaining agents regain a rigid formation. To illustrate the closing ranks problem, consider the following example: A minimally rigid formation is shown in Figure 3a. Let us assume that the agent indicated with an arrow departs from the formation (e.g., because of a failure or destruction) and this results in the formation shown in Figure 3b. Note that, as will be explained in $\S 2$, unless the departed agent has only $d$ links in $d$-space $(d=2,3)$, the formation with the remaining agents is not going to be rigid anymore. It is desirable that the formation regains rigidity.

One strategy to maintain rigidity is to create a rigid formation with the remaining agents by generating entirely new links between them. Although this is a viable solution, it has a disadvantage. There is no guarantee that any remaining agent preserve its links. Therefore, any agent, even one far away from the departed agent, may have to change its links.

Another strategy might be to create a new link between every pair of agents that had a link with the removed agent as shown in Figure 4a. This strategy avoids creating an entirely new set of links and also preserves all the remaining links. However, it does not preserve minimal rigidity. It requires $k(k-1) / 2$ new links when an agent with $k$ links departs. As will be explained in $\S 2$, the number of new links required for minimal rigidity is $k-2$ and $k-3$ in 2 - and 3-dimensional space respectively (Figure $4 \mathrm{~b})$.

The main goal of this section is to find strategies for the closing ranks problem. We take the following objectives into consideration in those strategies:

1. to regain "minimal" rigidity;

2. to preserve the remaining sensing and communication links after agent departure;

3. to insert the new links between only the neighbors of the departed agent.

The paper is organized as follows: In $\S 2$, we introduce an approach for creating 


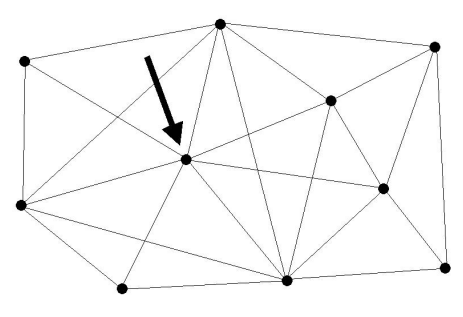

(a)

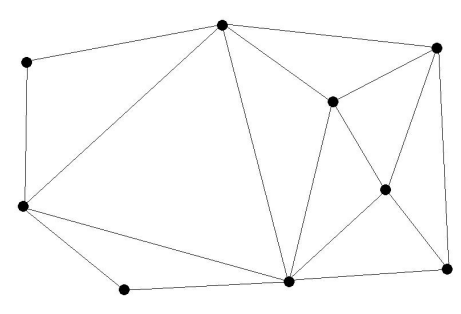

(b)

FIG. 3. a) The initial formation with the minimum number of sensing and communication links in 3-dimensional space. The agent to be removed is indicated with an arrow. (b) The remaining non-rigid formation after the agent departs.

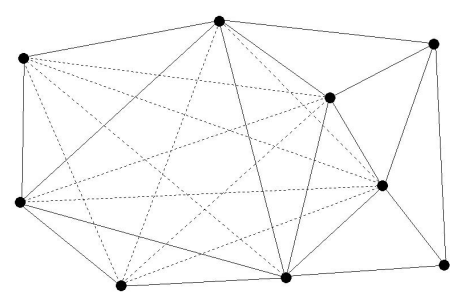

(a)

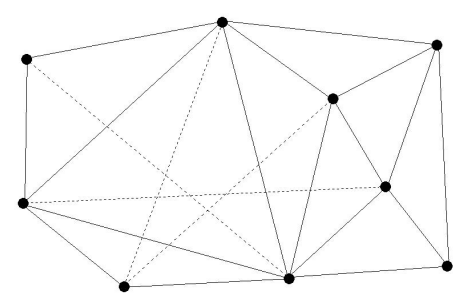

(b)

FIG. 4. a) One strategy of maintaining rigidity is to connect every pair of agents (dotted links) that were connected to the removed agent. (b) The four links chosen as depicted (dotted links) will suffice to regain rigidity.

sensor and network topologies of rigid formations with distance information between agents in 2- and 3-dimensional space [10]. Distances between all agents can be maintained fixed by directly measuring some distances between agents and keeping them at their desired values. We introduce such a strategy for maintaining formations with a limited number of links based on distances. The splitting and merging problems can be considered to be special cases of the "minimal cover problem", which we address in $\S 3$. To solve the minimal cover problem, we develop a novel procedure. This procedure can be used for creating minimally rigid post-split sub-formations from non-rigid post-split sub-formations and also for creating a minimally rigid postmerged formation from rigid pre-merged sub-formations, which are addressed in $\S 4$ and $\S 5$, respectively. In $\S 6$, we show that minimal rigidity can be regained in the closing ranks problem by inserting links between only the neighbors of the departed agent without making any changes in the set of links between the other remaining 
agents. We end the paper with summary and concluding remarks in $\S 7$.

2. Rigid Formations. We start with an overview of rigidity. Recall that a formation is rigid if the distance between each pair of agents does not change over time under ideal conditions. It is not necessary to have sensing and communication links between each pair of agents to maintain a rigid formation [10]. Distances between all agent pairs can be held fixed by directly measuring distances between only some agents and keeping them at desired values. We show such an approach for maintaining formations with a limited number of links with distance information both in 2- and 3-dimensional space.

Central to the development of the approach in this section will be rigid frameworks studied in mathematics and engineering for more than a century under different names such as frameworks, linkages, and mechanisms (see for example $[2,8,12,15,16,17,18$, $21,22,23,25])$. One way of visualizing rigidity is to imagine a collection of rigid bars connected to one another by idealized ball joints, which is called a bar-joint framework. By an idealized ball joint we mean a connection between a collection of bars which imposes only the restriction that the bars share common endpoints. Now, can the bars and joints be moved in a continuous manner without changing the lengths of any of the bars, where translations and rotations do not count? If so, the framework is non-rigid; if not, it is rigid. (Precise definitions will appear in the sequel.) The answer depends on factors such as which bars are connected to each other at which ball joints, bar lengths, and the dimensionality of the space in which the framework is placed. Actual physical bar-joint frameworks can be used in modeling a wide variety of physical structures, including rigid ones such as bridges as well as non-rigid structures such as organic molecules. Appropriate bar-joint frameworks representing such structures could be constructed to test the model for rigidity. However, such a concrete framework is feasible only for 2- and 3-dimensional space, and such concrete models become cumbersome as the number of bars and joints increases. The aim of rigidity theory is to develop methods for predicting rigidity without building a model.

In this section, essentially complete theory of rigid formations in 2-dimensional space is reviewed, as well as known partial results for 3-dimensional space. We review "generic" rigidity, which is the type of rigidity most useful for our purposes. In practice, actual agent groups cannot be expected to move exactly in rigid formation because of sensing, modeling, and actuation errors. With generic rigidity, the topology will be robust for maintaining formations under small perturbations. Although there is no existing comparable complete theory for 3-dimensional space, there are useful partial results $[27,28]$. We introduce sequential techniques to generate rigid classes of formations both in 2- and 3-dimensional space. The approach presented in this section forms the basis of the techniques developed in the subsequent sections. 
2.1. Point Formations and Rigidity. By a $d$-dimensional point formation at $p \triangleq$ column $\left\{p_{1}, p_{2}, \ldots, p_{n}\right\}$, written $\mathbb{F}_{p}$, is meant a set of $n$ points $\left\{p_{1}, p_{2}, \ldots, p_{n}\right\}$ in $\mathbb{R}^{d}$ together with a set $\mathcal{L}$ of $k$ maintenance links, labelled $(i, j)$, where $i$ and $j$ are distinct integers in $\{1,2, \ldots, n\}$; the length of link $(i, j)$ is the Euclidean distance between point $p_{i}$ and $p_{j}$. The idea of a point formation is essentially the same as the concept of a "framework" studied in mathematics [22, 27, 25] as well as within the theory of structures in mechanical and civil engineering. For our purposes, a point formation $\mathbb{F}_{p}=\left(\left\{p_{1}, p_{2}, \ldots, p_{n}\right\}, \mathcal{L}\right)$ provides a natural high-level model for a set of $n$ agents moving in real 2- or 3 - dimensional space. In this context, the points $p_{i}$ represent the positions of agents in $\mathbb{R}^{d}\{d=2$ or 3$\}$ and the links in $\mathcal{L}$ label those specific agent pairs whose inter-agent distances are to be maintained over time. In practice actual agent positions cannot be expected to move exactly in formation because of sensing errors, vehicle modelling errors, etc. The ideal benchmark formation against which the performance of an actual agent formation is to be measured is called a reference formation.

Each point formation $\mathbb{F}_{p}$ uniquely determines a graph $\mathbb{G} \triangleq(\mathcal{V}, \mathcal{L})$ with vertex set $\mathcal{V} \triangleq\{1,2, \ldots, n\}$ and edge set $\mathcal{L}$, as well as a distance function $\delta: \mathcal{L} \rightarrow \mathbb{R}$ whose value at $(i, j) \in \mathcal{L}$ is the distance between $p_{i}$ and $p_{j}$. Let us note that the distance function of $\mathbb{F}_{p}$ is the same as the distance function of any point formation $\mathbb{F}_{q}$ with the same graph as $\mathbb{F}_{p}$ provided $q$ is congruent to $p$ in the sense that there is a distance preserving map $T: \mathbb{R}^{d} \rightarrow \mathbb{R}^{d}$ such that $T\left(q_{i}\right)=p_{i}, i \in\{1,2, \ldots, n\}$. In the sequel we will say that two point formations $\mathbb{F}_{p}$ and $\mathbb{F}_{q}$ are congruent if they have the same graph and if $q$ and $p$ are congruent.

By a trajectory of $\mathbb{F}_{p}$, we mean a continuously parameterized, one-parameter family of points $\{q(t): t \geq 0\}$ in $\mathbb{R}^{n d}$, which contains $p$. A point formation $\mathbb{F}_{p}$ is said to be rigid if the distance between every pair of its points remains constant along any trajectory on which the lengths of all of its maintenance links in $\mathcal{L}$ are kept fixed. Alternatively, we can define a rigid point formation as follows: A formation is said to undergo rigid motion along a trajectory $q([0, \infty)) \triangleq\left\{\operatorname{column}\left\{q_{1}(t), q_{2}(t), \ldots, q_{n}(t)\right\}\right.$ : $t \geq 0\}$ if the Euclidean distance between each pair of points $q_{i}(t)$ and $q_{j}(t)$ remains constant all along the trajectory. Let us note that $\mathbb{F}_{p}$ undergoes rigid motion along a trajectory $q([0, \infty))$ just in case each pair of points $q\left(t_{1}\right), q\left(t_{2}\right) \in q([0, \infty))$ are congruent. The set of points $\mathcal{M}_{p}$ in $\mathbb{R}^{n d}$ which are congruent to $p$ is known to be a smooth manifold. It is clear that any trajectory along which $\mathbb{F}_{p}$ undergoes rigid motion must lie completely within $\mathcal{M}_{p}$; conversely any trajectory of $\mathbb{F}_{p}$ that lies within $\mathcal{M}_{p}$ is one along which $\mathbb{F}_{p}$ undergoes rigid motion. A point formation $\mathbb{F}_{p}$ is said to be rigid if rigid motion is the only kind of motion it can undergo along any trajectory on which the lengths of all links in $\mathcal{L}$ remain constant. Thus, if $\mathbb{F}_{p}$ is rigid, it is possible to "keep formation" by making sure that the lengths of the formation's maintained links do not change as the formation moves. 
A graph is called complete if each pair of its vertices is connected by an edge. The definition of rigid formation implies that every formation $\mathbb{F}_{p}$ whose graph $\mathbb{G}$ is complete is automatically rigid. The converse, however, is not generally true.

Whether a given point formation is rigid or not can be studied by examining what happens to the given point formation $\mathbb{F}_{p}=\left(\left\{p_{1}, p_{2}, \ldots, p_{n}\right\}, \mathcal{L}\right)$ with $m$ maintenance links, along the trajectory $q([0, \infty)) \triangleq\left\{\left\{q_{1}(t), q_{2}(t), \ldots, q_{n}(t)\right\}: t \geq 0\right\}$ on which the Euclidean distances $d_{i j} \triangleq\left\|p_{i}-p_{j}\right\|$ between pairs of points $\left(p_{i}, p_{j}\right)$ for which $(i, j)$ is a link are constant. Along such a trajectory

$$
\left(q_{i}-q_{j}\right) \cdot\left(q_{i}-q_{j}\right)=d_{i j}^{2}, \quad(i, j) \in \mathcal{L}, \quad t \geq 0
$$

We note that the existence of a trajectory is equivalent to the existence of a piecewise analytic path, with all derivatives at the initial point [23]. It is also equivalent to the existence of a sequence of formations on $p(n), n=1,2, \ldots$ with the same measurements, and with $\lim _{n \rightarrow \infty} p(n)$ converging to $p$. Assuming a smooth (piecewise analytic) trajectory, we can differentiate to get

$$
\left(q_{i}-q_{j}\right) \cdot\left(\dot{q}_{i}-\dot{q}_{j}\right)=0, \quad(i, j) \in \mathcal{L}, \quad t \geq 0
$$

Here, $\dot{q}_{i}$ is the velocity of point $i$. The $m$ equations can be collected into a single matrix equation

$$
R(q) \dot{q}=0,
$$

where $\dot{q}=$ column $\left\{\dot{q}_{1}, \dot{q}_{2}, \ldots, \dot{q}_{n}\right\}$ and $R(q)$ is a specially structured $m \times d n$ matrix called the rigidity matrix $[22,27,28]$.

EXAmple 1. Consider a planar point formation $\mathbb{F}_{p}$ shown in Figure 5. This has a rigidity matrix as follows:

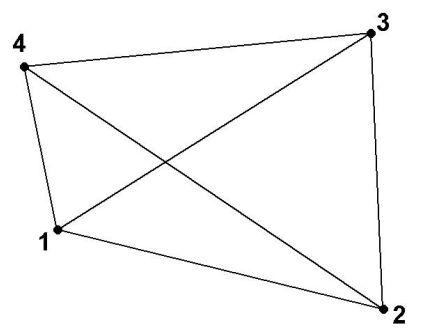

FIG. 5. A planar point formation. 


\begin{tabular}{|c|cc|cc|cc|cc|}
\hline$R(p)$ & \multicolumn{2}{|c|}{$v_{1}$} & \multicolumn{2}{c|}{$v_{2}$} & \multicolumn{2}{c|}{$v_{3}$} & \multicolumn{2}{c|}{$v_{4}$} \\
\hline$(1,2)$ & $x_{1}-x_{2}$ & $y_{1}-y_{2}$ & $x_{2}-x_{1}$ & $y_{2}-y_{1}$ & 0 & 0 & 0 & 0 \\
\hline$(1,3)$ & $x_{1}-x_{3}$ & $y_{1}-y_{3}$ & 0 & 0 & $x_{3}-x_{1}$ & $y_{3}-y_{1}$ & 0 & 0 \\
\hline$(1,4)$ & $x_{1}-x_{4}$ & $y_{1}-y_{4}$ & 0 & 0 & 0 & 0 & $x_{4}-x_{1}$ & $y_{4}-y_{1}$ \\
\hline$(2,3)$ & 0 & 0 & $x_{2}-x_{3}$ & $y_{2}-y_{3}$ & $x_{3}-x_{2}$ & $y_{3}-y_{2}$ & 0 & 0 \\
\hline$(2,4)$ & 0 & 0 & $x_{2}-x_{4}$ & $y_{2}-y_{4}$ & 0 & 0 & $x_{4}-x_{2}$ & $y_{4}-y_{2}$ \\
\hline$(3,4)$ & 0 & 0 & 0 & 0 & $x_{3}-x_{4}$ & $y_{3}-y_{4}$ & $x_{4}-x_{3}$ & $y_{4}-y_{3}$ \\
\hline
\end{tabular}

Because any trajectory of $\mathbb{F}_{p}$ which lies within $\mathcal{M}_{p}$, is one along which $\mathbb{F}_{p}$ undergoes rigid motion, (2) automatically holds along any trajectory which lies within $\mathcal{M}_{p}$. From this, it follows that the tangent space to $\mathcal{M}_{p}$ at $q$, written $\mathcal{T}_{q}$, must be contained in the kernel of $R(q)$. Since $p$ must be on any such trajectory, it must be true that $\mathcal{T}_{p} \subset$ kernel $R(p)$. If $\dot{q}$ satisfies (3), then it lies in the tangent space. If the affine span of the points $p_{1}, p_{2}, \ldots, p_{n}$ is $\mathbb{R}^{n}$ (which means that the points $p_{1}, p_{2}, \ldots, p_{n}$ do not lie on any hyperplane in $\left.\mathbb{R}^{n}\right)$, then $\mathcal{M}_{p}$ is $n(n+1) / 2$ dimensional since it arises from the $n(n-1) / 2$-dimensional manifold of orthogonal transformations of $\mathbb{R}^{n}$ and the $n$-dimensional manifold of translations of $\mathbb{R}^{n}[22]$. Thus $\mathcal{M}_{p}$ is 6-dimensional for $\mathbb{F}_{p}$ in $\mathbb{R}^{3}$, and 3 -dimensional for $\mathbb{F}_{p}$ in $\mathbb{R}^{2}$. We have $\operatorname{rank} R(q)=n d-\operatorname{dimension}(\operatorname{kernel}(R(q))) \leq n d-n(n+1) / 2$.

We have the following theorem $[2,12,22,28]$ :

TheOREM 2. Assume $\mathbb{F}_{p}$ is a formation with at least $d$ points in $d$-space $\{d=2$, or 3$\}$ where $\operatorname{rank} R(p)=\max \left\{\operatorname{rank} R(x): x \in \mathbb{R}^{d}\right\} . \mathbb{F}_{p}$ is rigid in $\mathbb{R}^{d}$ if and only if

$$
\operatorname{rank} R(p)= \begin{cases}2 n-3 & \text { if } d=2, \\ 3 n-6 & \text { if } d=3\end{cases}
$$

This theorem leads to the notion of the "generic" behavior of rigidity. When the rank is less than the maximum, the formation may still be rigid. However, this type of rigidity is unstable. For almost all small changes in the position of $p$ (or in the lengths of the maintenance links), the formation will no longer be rigid. We are interested in "generic rigidity", a property that will hold for all small changes in $p$.

2.2. Generic Rigidity. In this section, we define a type of rigidity, called "generic rigidity," that is more useful for our purposes. A point formation $\mathbb{F}_{p}$ is generically rigid if it is rigid for almost all choices of $p$ in $\mathbb{R}^{d n}$. It is possible to characterize generic rigidity in terms of the "generic rank" of $R$ where by $R$ 's generic or maximal rank we mean the largest value of $\operatorname{rank}\{R(q)\}$ as $q$ ranges over all values in $\mathbb{R}^{n d}$. The following theorem is due to Roth [22].

ThEOREM 3. A formation $\mathbb{F}_{p}$ with at least $d$ points in $d$-space $\{d=2$, or 3$\}$ is generically rigid if and only if

$$
\text { generic } \operatorname{rank}\{R\}= \begin{cases}2 n-3 & \text { if } d=2, \\ 3 n-6 & \text { if } d=3\end{cases}
$$


To understand this type of rigidity, it is useful to observe that the set of points $p$ that satisfy the condition $\operatorname{rank} R(p)=\max \left\{\operatorname{rank} R(x): x \in \mathbb{R}^{d}\right\}$ is a dense open subset of $\mathbb{R}^{\text {nd }}[22]$. Generic rigidity is a property of only the set of maintenance links, or the underlying graph. It does not even claim that $\mathbb{F}_{p}$ itself is rigid but only that almost all nearby points $q$ give rigid formations $\mathbb{F}_{q}$. The concept of generic rigidity does not depend on the precise distances between the points of $\mathbb{F}_{p}$ but examines how well the rigidity of formations can be judged by knowing the vertices and their incidences, in other words, by knowing the underlying graph. A point formation $\mathbb{F}_{p}$ is strongly generically rigid if it is generically rigid and if $\operatorname{rank} R(p)=$ generic $\operatorname{rank}\{R\}$. Hence, a strongly generically rigid point formation is rigid and it remains rigid under small perturbations. For this reason, it is a desirable specialization of the concept of a "rigid formation" for our purposes. We have the following theorem for a strongly generically rigid point formation and a generically rigid graph [27]:

THEOREM 4. For a formation $\mathbb{F}_{p}$ in d-space with at least $d$ points, the following are equivalent:

1. the formation's underlying graph $\mathbb{G}=(\mathcal{V}, \mathcal{L})$ is generically rigid in d-dimensional space $(d=2,3)$;

2. for some $p$,

$$
\operatorname{rank}\{R(p)\}= \begin{cases}2 n-3 & \text { if } d=2, \\ 3 n-6 & \text { if } d=3\end{cases}
$$

3. for almost all $p$, the formation $\mathbb{F}_{p}$ is strongly generically rigid.

The generic rigidity question can be reduced to developing a test for computing the generic rank of a matrix. The generic rank question is closely related to the question of structural controllability treated some time ago by Corfmat and Morse [7] and Anderson and Hong [1]. In particular, in Anderson and Hong [1], explicit constructions are given for determining the generic rank of an arbitrary matrix net of the form

$$
M(z)=M_{0}+z_{1} M_{1}+\cdots+z_{n} M_{n}, \quad M_{i} \in \mathbb{R}^{n \times n}
$$

over the linear space of all real $n$-vectors $z=$ column $\left\{z_{1}, z_{2}, \cdots, z_{n}\right\}, z_{i} \in \mathbb{R}$. Unfortunately these constructions are computationally complex and reveal little about the structure of generically rigid formations.

As noted above, the concept of generic rigidity does not depend on the precise distances between the points in $\mathbb{F}_{p}$. For 2-dimensional space, we have a complete combinatorial characterization of generically rigid graphs, which was first proved by Laman in 1970 [17]. In the theorem below, |.| is used to denote the cardinal number of a set, i.e., the number of elements in a set. 
Theorem 5 (Laman [17]). A graph $\mathbb{G}=(\mathcal{V}, \mathcal{L})$ (where $\mathcal{L} \neq \emptyset$ or $n>1$ ) is generically rigid in 2-dimensional space if and only if there is a subset $\mathcal{L}^{\prime} \subseteq \mathcal{L}$ satisfying the following two conditions: (1) $\left|\mathcal{L}^{\prime}\right|=2|\mathcal{V}|-3$, (2) For all $\mathcal{L}^{\prime \prime} \subseteq \mathcal{L}^{\prime}, \mathcal{L}^{\prime \prime} \neq \emptyset,\left|\mathcal{L}^{\prime \prime}\right| \leq$ $2\left|\mathcal{V}\left(\mathcal{L}^{\prime \prime}\right)\right|-3$, where $\left|\mathcal{V}\left(\mathcal{L}^{\prime \prime}\right)\right|$ is the number of vertices that are end-vertices of the edges in $\mathcal{L}^{\prime \prime}$.

There is no comparable complete result for 3-dimensional space, though there are useful partial results $[27,28]$. So one has to place randomly chosen entries in the rigidity matrix and examine the rigidity matrix in 3 -dimensional space. The generalization of the Laman counts: (1) $\left|\mathcal{L}^{\prime}\right|=3 n-6$, (2) for all $\mathcal{L}^{\prime \prime} \subseteq \mathcal{L}^{\prime}, \mathcal{L}^{\prime \prime} \neq \emptyset,\left|\mathcal{L}^{\prime \prime}\right| \leq 3\left|\mathcal{V}\left(\mathcal{L}^{\prime \prime}\right)\right|-6$ are clearly necessary, where $\left|\mathcal{V}\left(\mathcal{L}^{\prime \prime}\right)\right|$ is the number of vertices that are end-vertices of the edges in $\mathcal{L}^{\prime \prime}$. However, as the graph in Figure 6 illustrates, these counts are not sufficient [27]. One can verify that this graph satisfies the generalization of the Laman counts. Nevertheless, it is not rigid since the two "bananas" can twist about the axis passing through their two end-points. Although we lack a characterization in 3 -dimensional space, there are sequential techniques to generate rigid classes of graphs both in 2- and 3-dimensional space based on the vertex addition, edge splitting and vertex splitting operations $[3,16,25,26,27]$. Some of these sequential results from the $19^{\text {th }}$ century are summarized in [16] and all are proven in $[25,26]$. We explain these techniques in the sequel, but before that, we discuss minimal rigidity in the next section.

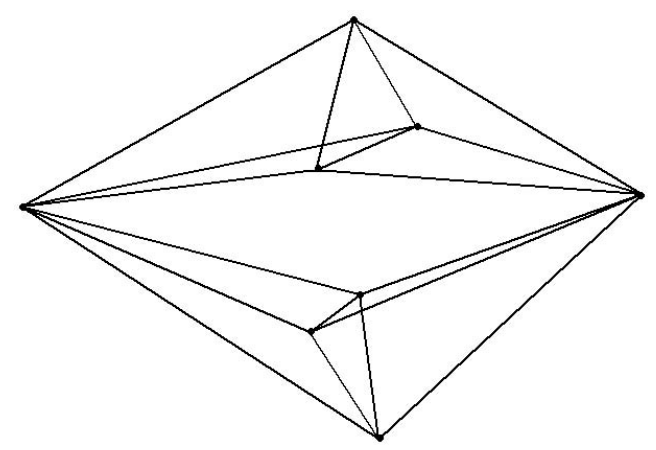

FIG. 6. This graph is not rigid since the two "bananas" can twist about the axis passing through their two end-points. On the other hand it can be verified that it satisfies Laman's conditions, with (1) $\left|\mathcal{L}^{\prime}\right|=3 n-6$, (2) For all $\mathcal{L}^{\prime \prime} \subseteq \mathcal{L}^{\prime}, \mathcal{L}^{\prime \prime} \neq \emptyset,\left|\mathcal{L}^{\prime \prime}\right| \leq 3\left|\mathcal{V}\left(\mathcal{L}^{\prime \prime}\right)\right|-6$.

2.3. Minimal Rigidity. A dependence on the maintenance link set of a point formation $\mathbb{F}_{p}=\left(\left\{p_{1}, p_{2}, \ldots, p_{n}\right\}, \mathcal{L}\right)$ is an assignment $\lambda: \mathcal{L} \rightarrow \mathbb{R}$, with $\lambda(i, j)=\lambda_{i, j}=$ $\lambda_{j, i}$, such that for each vertex $i$ :

$$
\sum_{j \mid(i, j) \in \mathcal{L}} \lambda_{i j}\left(p_{i}-p_{j}\right)=0
$$


This equation gives a row dependence of the rigidity matrix. A point formation is independent if its maintenance link set is independent as rows of the rigidity matrix. The scalars $\lambda_{i, j}$ are known as equilibrium stresses or self-stresses in structural engineering and in the mathematical theory of rigidity. A point formation is minimally rigid if removing any link makes it non-rigid. There are $2 n-3$ and $3 n-6$ maintenance links in minimally rigid formations in 2- and 3-dimensional space respectively. A graph is called (generically) ${ }^{1}$ minimally rigid in $d$-space if it is rigid and has exactly $d n-\left(\begin{array}{c}d+1 \\ 2\end{array}\right)$ edges. Minimally rigid point formations are also maximally independent point formations, corresponding to bases in vector spaces as minimally spanning sets and maximally independent sets.

If a point formation is rigid but not minimally rigid, we say that there is redundancy in the link set $\mathcal{L}$. Let us suppose that a link $(i, j)$ is removed from a rigid point formation. If the formation remains rigid then $(i, j)$ is called a redundant link in the initial formation (redundant edge in the underlying graph). If adding a link $(i, j)$ does not increase the rank of the rigidity matrix, then we call $(i, j)$ an implicit link (implicit edge in the underlying graph).

In 2-dimensional space, a set of edges $\mathcal{L}$ is called a set of independent edges or an independent edge set if $|\mathcal{L}| \leq 2|\mathcal{V}(\mathcal{L})|-3$ and for every $\mathcal{L}^{\prime} \subseteq \mathcal{L},\left|\mathcal{L}^{\prime}\right| \leq 2\left|\mathcal{V}\left(\mathcal{L}^{\prime}\right)\right|-3$. If the set of edges $\mathcal{L}$ of a planar graph $\mathbb{G}=(\mathcal{V}, \mathcal{L})$ is independent, then $\mathbb{G}=(\mathcal{V}, \mathcal{L})$ is called an independent graph. The two conditions in Laman's Theorem check whether there are at least $2 n-3$ independent edges and whether the edges are distributed in such a way that there are no redundancies.

Example 6. Consider the planar point formation $\mathbb{F}_{p}$ in Example 1. This has a rigidity matrix with the column for row dependence $\lambda$ as follows:

\begin{tabular}{|c|cc|cc|cc|cc|c|}
\hline$R\left(\mathbb{F}_{p}\right)$ & \multicolumn{2}{|c|}{$v_{1}$} & \multicolumn{2}{c|}{$v_{2}$} & \multicolumn{2}{c|}{$v_{3}$} & \multicolumn{2}{c|}{$v_{4}$} & $\lambda$ \\
\hline$(1,2)$ & $x_{1}-x_{2}$ & $y_{1}-y_{2}$ & $x_{2}-x_{1}$ & $y_{2}-y_{1}$ & 0 & 0 & 0 & 0 & $\lambda_{12}$ \\
\hline$(1,3)$ & $x_{1}-x_{3}$ & $y_{1}-y_{3}$ & 0 & 0 & $x_{3}-x_{1}$ & $y_{3}-y_{1}$ & 0 & 0 & $\lambda_{13}$ \\
\hline$(1,4)$ & $x_{1}-x_{4}$ & $y_{1}-y_{4}$ & 0 & 0 & 0 & 0 & $x_{4}-x_{1}$ & $y_{4}-y_{1}$ & $\lambda_{14}$ \\
\hline$(2,3)$ & 0 & 0 & $x_{2}-x_{3}$ & $y_{2}-y_{3}$ & $x_{3}-x_{2}$ & $y_{3}-y_{2}$ & 0 & 0 & $\lambda_{23}$ \\
\hline$(2,4)$ & 0 & 0 & $x_{2}-x_{4}$ & $y_{2}-y_{4}$ & 0 & 0 & $x_{4}-x_{2}$ & $y_{4}-y_{2}$ & $\lambda_{24}$ \\
\hline$(3,4)$ & 0 & 0 & 0 & 0 & $x_{3}-x_{4}$ & $y_{3}-y_{4}$ & $x_{4}-x_{3}$ & $y_{4}-y_{3}$ & $\lambda_{34}$ \\
\hline
\end{tabular}

If we consider a particular point formation $p_{1}=(0,0), p_{2}=(2,0), p_{3}=(3,2)$, and $p_{4}=(1,1)$, then it can be verified that the assignment $\lambda$ given in the final column is a row dependence.

\footnotetext{
${ }^{1}$ In the sequel, we use the term rigid graph instead of generically rigid graph unless there is a danger of confusion.
} 


\begin{tabular}{|c|c|c|c|c|c|c|c|c|c|}
\hline$R\left(\mathbb{F}_{p}\right)$ & \multicolumn{2}{|c|}{$v_{1}$} & \multicolumn{2}{|c|}{$v_{2}$} & \multicolumn{2}{|c|}{$v_{3}$} & \multicolumn{2}{|c|}{$v_{4}$} & $\lambda$ \\
\hline$(1,2)$ & -2 & 0 & 2 & 0 & 0 & 0 & 0 & 0 & $\frac{3}{2}$ \\
\hline$(1,3)$ & -3 & -2 & 0 & 0 & 3 & 2 & 0 & 0 & -3 \\
\hline$(1,4)$ & -1 & -1 & 0 & 0 & 0 & 0 & 1 & 1 & 6 \\
\hline$(2,3)$ & 0 & 0 & -1 & -2 & 1 & 2 & 0 & 0 & 1 \\
\hline$(2,4)$ & 0 & 0 & 1 & -1 & 0 & 0 & -1 & 1 & -2 \\
\hline$(3,4)$ & 0 & 0 & 0 & 0 & 2 & 1 & -2 & -1 & 4 \\
\hline
\end{tabular}

2.4. Sequential Techniques. In this section, we present sequential techniques to create minimally rigid point formations. As noted earlier, Laman's Theorem characterizes rigidity in 2-dimensional space. But there is no comparable complete result for 3-dimensional space. Although we lack a characterization in 3-dimensional space, there are sequential techniques for generating rigid classes of graphs both in 2- and 3 -dimensional space based on what are known as the vertex addition, edge splitting, and vertex splitting operations. First, we introduce the first two of these three operations, namely the vertex addition and edge splitting operations. Then we present sequences to create rigid point formations in which these operations are used. Before explaining these operations and sequences, we introduce some additional terminology.

If $(i, j)$ is an edge, then we say that $i$ and $j$ are adjacent or that $j$ is a neighbor of $i$ and $i$ is a neighbor of $j .{ }^{2}$ The vertices $i$ and $j$ are incident with the edge $(i, j)$. Two edges are adjacent if they have exactly one common end-vertex. The degree or valency of a vertex $i$ is the number of neighbors of $i$. If a vertex has $k$ neighbors, it is called a vertex of degree $k$ or a $k$-valent vertex. We refer to Bollobás [6], and Godsil and Royle [13] for a detailed treatment of graph theoretic terms.

One operation is the vertex addition: given a minimally rigid graph $\mathbb{G}=(\mathcal{V}, \mathcal{L})$, we add a new vertex $i$ with $d$ edges between $i$ and $d$ other vertices in $\mathcal{V}$. The other is the edge splitting: given a minimally rigid graph $\mathbb{G}=(\mathcal{V}, \mathcal{L})$, we remove an edge $(j, k)$ in $\mathcal{L}$ and then we add a new vertex $i$ with $d+1$ edges by inserting two edges $(i, j),(i, k)$ and $d-1$ edges between $i$ and $d-1$ vertices (other than $j, k)$ in $\mathcal{V}$.

Now we are ready to present the following theorems:

TheOREM 7 (vertex addition [25]). Let $\mathbb{G}=(\mathcal{V}, \mathcal{L})$ be a graph with a vertex $i$ of degree $d$ in d-space; let $\mathbb{G}^{*}=\left(\mathcal{V}^{*}, \mathcal{L}^{*}\right)$ denote the subgraph obtained by deleting $i$ and the edges incident with it. Then $G$ is generically minimally rigid if and only if $\mathbb{G}^{*}$ is generically minimally rigid.

Theorem 8 (edge splitting [25]). Let $\mathbb{G}=(\mathcal{V}, \mathcal{L})$ be a graph with a vertex $i$ of degree $d+1$; let $\mathcal{V}_{i}$ be the set of vertices incident to $i$; and let $\mathbb{G}^{*}=\left(\mathcal{V}^{*}, \mathcal{L}^{*}\right)$ be the subgraph obtained by deleting $i$ and its $d+1$ incident edges. Then $\mathbb{G}$ is generically minimally rigid if and only if there is a pair $j, k$ of vertices of $\mathcal{V}_{i}$ such that the edge

\footnotetext{
${ }^{2}$ The neighbor relation is symmetric throughout the paper, i.e., if agent $i$ senses or communicates with agent $j$, so does agent $j$ with agent $i$.
} 
$(j, k)$ is not in $\mathcal{L}^{*}$ and the graph $\mathbb{G}^{\prime}=\left(\mathcal{V}^{*}, \mathcal{L}^{*} \bigcup(j, k)\right)$ is generically minimally rigid.

These two operations are used in Henneberg sequences.

Henneberg Sequences:. Henneberg sequences are a systematic way of generating minimally rigid graphs based on the vertex addition and edge splitting operations [25]. In $d$-space, we are given a sequence of graphs: $\mathbb{G}_{d}, \mathbb{G}_{d+1}, \ldots, \mathbb{G}_{|\mathcal{V}|}$ such that:

1. $\mathbb{G}_{d}$ is the complete graph on $d$ vertices;

2. $\mathbb{G}_{i+1}$ comes from $\mathbb{G}_{i}$ by adding a new vertex either by (i) the vertex addition or (ii) the edge splitting operation.

Note that $\mathbb{G}_{i}$ and $\mathbb{G}_{i+1}$ correspond to $\mathbb{G}^{*}$ and $\mathbb{G}$ in the statements of Theorem 7 and Theorem 8. All graphs in the sequence are minimally rigid in $d$-space. Figure 7 depicts such a Henneberg sequence in 3-dimensional space.

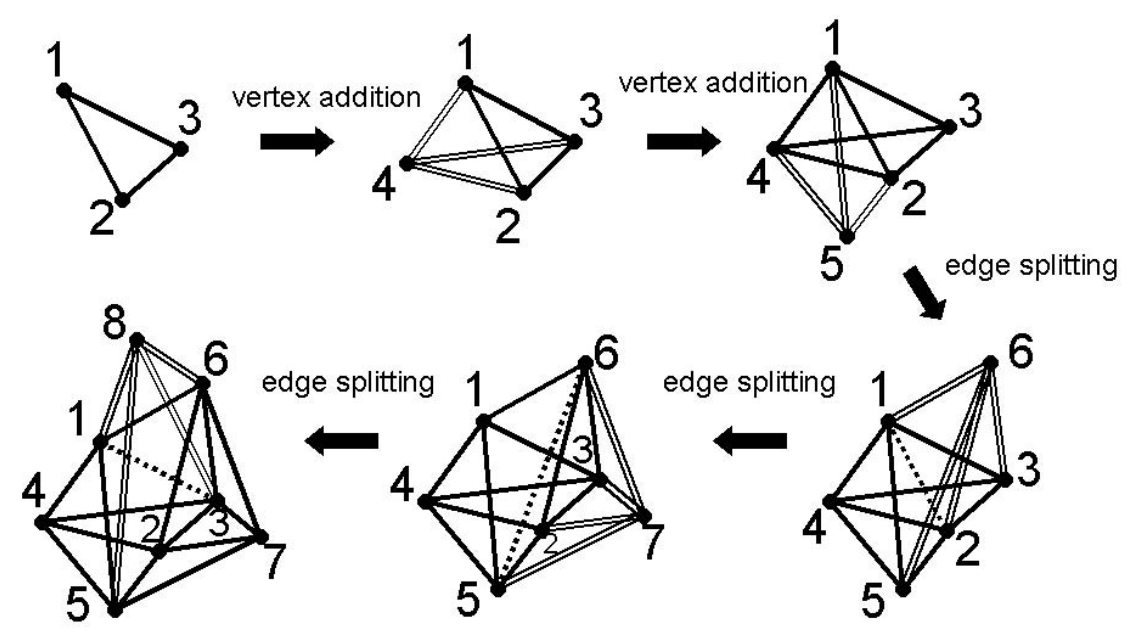

FIG. 7. A rigid point formation generated by a Henneberg sequence in 3-dimensional space. Double-lined edges indicate edges created for new vertices. Dashed edges indicate removed edges in the edge splitting operation.

In 2-dimensional space, the two operations of vertex addition and edge splitting are sufficient to generate all minimally rigid graphs starting from a single edge [25]. In 3-dimensional space, they generate a proper subclass of minimally rigid graphs starting from a triangle. The reason behind this difference between planar and spatial cases is the following observation.

A minimally rigid graph in 2-dimensional space may have all vertices of degree larger than $2 ;|\mathcal{L}|=2|\mathcal{V}|-3$ or equivalently $2|\mathcal{L}|=4|\mathcal{V}|-6$ guarantees that some vertices have degree 3 or less. If $\mathbb{G}=(\mathcal{V}, \mathcal{L})$ is a graph with $|\mathcal{V}|$ vertices which is minimally rigid, the existence of at least one vertex of degree 2 or 3 means that by Theorem 7 or Theorem 8 there exists a minimally rigid graph $\mathbb{G}^{*}$ with $|\mathcal{V}|-1$ vertices, and one can go from $\mathbb{G}$ to $\mathbb{G}^{*}$ by the vertex addition or edge splitting operations. 
Then one uses the same procedure on $\mathbb{G}^{*}$. Hence, all minimally rigid graphs can be generated by the vertex addition and edge splitting operations alone. It is also true that starting with a single edge only minimally rigid graphs are generated with these operations in 2-dimensional space.

On the other hand, a minimally rigid graph in 3-dimensional space may have all vertices of degree larger than $4 ;|\mathcal{L}|=3|\mathcal{V}|-6$ or equivalently $2|\mathcal{L}|=6|\mathcal{V}|-12$ guarantees only that some vertices have degree 5 or less. A quick check with the vertex addition and edge splitting operations in 3-dimensional space tells us that we can generate vertices of degree 3 and 4 with these operations, but not of degree 5 . We need other types of operations to generate minimally rigid graphs in 3-dimensional space with all vertices having a degree of 5 or higher, and to remove a vertex of degree 5 from a minimally rigid graph in 3-dimensional space. For example, the graph of an icosahedron shown in Figure 8 is minimally rigid and has all vertices of degree 5 [27].

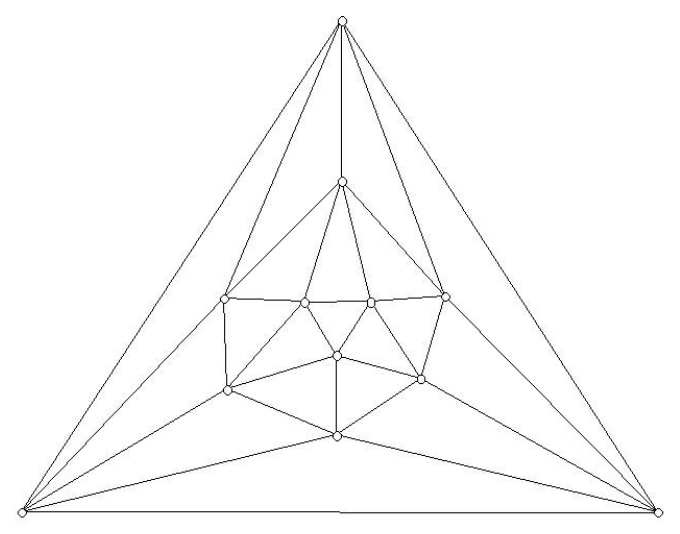

FIG. 8. The graph of an icosahedron is minimally rigid and has all vertices of degree 5 .

The following theorem is about removing a 5 -valent vertex in a minimally rigid graph.

Theorem 9 (Removing a 5-valent vertex $[25])$. Let $\mathbb{G}=(\mathcal{V}, \mathcal{L})$ be a minimally rigid graph with a 5-valent vertex a and edges $\left(a, b_{i}\right), 1 \leq i \leq 5$. Let $\mathbb{G}^{*}=\left(\mathcal{V}^{*}, \mathcal{L}^{*}\right)$ be a graph obtained by removing vertex $a$ and the edges $\left(a, b_{i}\right), 1 \leq i \leq 5$ from $\mathbb{G}$. Then one of the following is true (see Figure 9):

1. for some choice of two non-adjacent edges with vertices drawn from $b_{1}, b_{2}, \ldots$, $b_{5}$, the graph obtained by inserting these edges is minimally rigid in 3-dimensional space.

2. for two choices of adjacent pairs of edges with vertices drawn from $b_{1}, b_{2}, \ldots$, $b_{5}$ (not all adjacent with a single vertex), the two graphs obtained from $\mathbb{G}^{*}$ by inserting these pairs are both minimally rigid in 3-dimensional space. 
Adding a 5-valent vertex to a minimally rigid graph in 3-dimensional space to guarantee preservation of minimal rigidity is a long-standing problem. So far there are only partial results $[14,24,25]$. There are two sequential operations for adding 5 -valent vertices to minimally rigid graphs. They are conjectured to preserve minimal rigidity in 3-dimensional space. The first operation is replacing an $\mathrm{X}$ (i.e., two edges that do not share any vertices) by a vertex of degree five by connecting the vertex to the end vertices of these two edges plus an additional vertex. The second operation is the double $\mathrm{V}$ replacement. This operation takes two graphs $\mathbb{G}_{1}$ and $\mathbb{G}_{2}$ that are minimally rigid in 3-dimensional space to a graph $\mathbb{G}$ that is generically rigid in 3dimensional space.

Conjecture 10 (Adding a 5-valent vertex by replacing a single $\mathrm{X}[25]$ ). If $\mathbb{G}=$ $(\mathcal{V}, \mathcal{L})$ is a minimally rigid graph in 3-dimensional space containing edges $(a, b),(c, d)$, then the graph obtained by deleting these two edges and adding a 5-valent vertex $i$ attached to the vertices $a, b, c, d$ and another vertex $e \in \mathcal{V}$ is minimally rigid in 3dimensional space (see Figure 9a).

Conjecture 11 (Adding a 5-valent vertex by replacing 2 V's [25]). If $\mathbb{G}_{1}=\mathbb{G} \cup$ $\{(a, b),(b, c)\}$ and $\mathbb{G}_{2}=\mathbb{G} \cup\{(\tilde{a}, \tilde{b}),(\tilde{b}, \tilde{c})\}$ are minimally rigid graphs in 3-dimensional space with $b \neq \tilde{b}$, then the graph $\mathbb{G}^{*}$ obtained from $\mathbb{G}$ by adding a 5-valent vertex $i$, attached to vertices including $a, b, c, \tilde{a}, \tilde{b}, \tilde{c}$, is also a minimally rigid graph in 3dimensional space (see Figure 9b).

Every minimally rigid graph can be generated by this extended Henneberg sequence, which includes the two operations in the conjectures with the vertex addition and the simpler edge splitting operations. What is unproven is that only minimally rigid graphs in 3-dimensional space are generated in this way. The lack of a simpler technique for adding 5 -valent vertices is connected to the failure of any simple spatial analogues of Laman's Theorem. As explained in $\S 2.2$, the graph in Figure 6 is an example of this failure.

There are two partial results which give sufficient conditions for the replacement of two edges by a 5 -valent vertex and three edges by a 6 -valent vertex. These operations are called vertex splitting. These operations can be used to create triangulated spheres, which are minimally rigid in 3-dimensional space and which have nice local properties in rigidity. We refer the reader to Gluck [12] and Eren [9] for further information on triangulated spheres.

Vertex Splitting: If the graph $\mathbb{G}^{\prime}$ is a vertex split of a generically rigid graph $\mathbb{G}$ on $d$ edges in $d$-space or a vertex split on $d-1$ edges, then $\mathbb{G}^{\prime}$ is generically rigid in $d$-space [26]. Vertex 3-splits on two edges and three edges are depicted in Figures 10a and 10b. By vertex $d$-split, we mean a split in $d$-space.

3. The Minimal Cover Problem. This section focuses on the "minimal cover problem." Before giving the definition of the minimal cover problem, we state our 


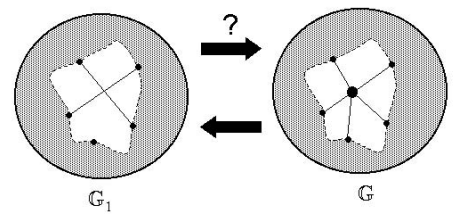

(a)

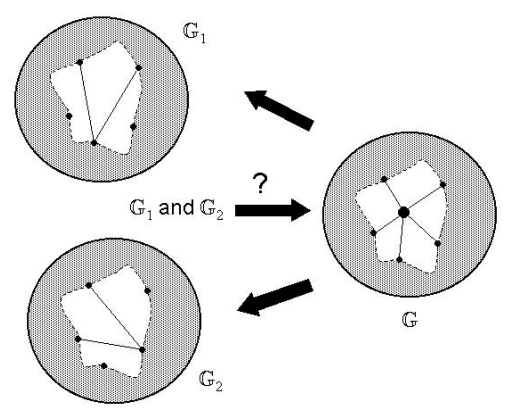

(b)

FIG. 9. (a) Replacing a vertex of degree 5 with an $X$ or vice versa in a minimally rigid formation; (b) Replacing a vertex of degree 5 with 2 V's or vice versa in a minimally rigid formation.

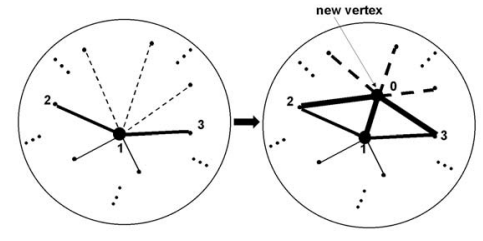

(a)

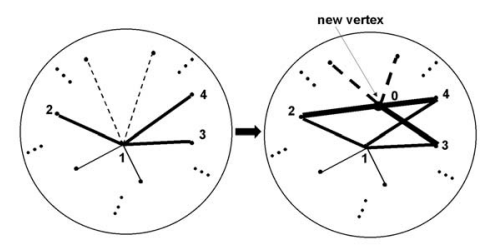

(b)

FIG. 10. (a) Vertex 3-split on two edges. Edges which are shifted to the new vertex 0 are shown with dashed edges, and new edges after split are shown with wider edges. (b) Vertex 3-split on three edges. Edges which are shifted to the new vertex 0 are shown with dashed edges, and new edges after split are shown with wider edges.

assumption.

Assumption: Let $\mathbb{G}=(\mathcal{V}, \mathcal{L})$ represent the underlying graph of each post-split formation in the splitting problem in $d$-space $(d=2,3)$, and the union of the underlying graphs of pre-merged sub-formations in the merging problem in $d$-space $(d=2,3)$. In splitting, we assume that $\mathbb{G}=(\mathcal{V}, \mathcal{L}) \subseteq \overline{\mathbb{G}}=(\overline{\mathcal{V}}, \overline{\mathcal{L}})$, where $\overline{\mathbb{G}}=(\overline{\mathcal{V}}, \overline{\mathcal{L}})$ is a graph created by a Henneberg sequence in $d$-space $(d=2,3)$ as explained in $\S 2.2$. In splitting, $\overline{\mathbb{G}}$ refers to the pre-split rigid formation created by a Henneberg sequence. In merging, we assume that each pre-merged rigid subgraph is created by a Henneberg sequence.

We note that the assumption is not a limitation on pre-split and pre-merged graphs in 2-dimensional space since there is a Henneberg sequence for all minimally rigid graphs in 2-dimensional space. However, the assumption is a limitation on pre-split and pre-merged graphs in 3-dimensional space since it is currently unknown 
whether there is a Henneberg sequence for all minimally rigid graphs in 3-dimensional space as explained in $\S 2.4$.

The minimal cover problem is to find a set of new edges to be inserted into graph $\mathbb{G}=(\mathcal{V}, \mathcal{L})$ so that the resulting graph $\mathbb{G}^{*}=\left(\mathcal{V}, \mathcal{L}^{*}\right)$ after insertions, is minimally rigid. Note that $\mathbb{G}$ and $\mathbb{G}^{*}$ have the same vertex set. We have the following lemma.

Lemma 12. The edge set of a graph $\mathbb{G}=(\mathcal{V}, \mathcal{L})$ that satisfy the assumption of the minimal cover problem is a set of independent edges.

Proof. In splitting, since the graph $\overline{\mathbb{G}}=(\overline{\mathcal{V}}, \overline{\mathcal{L}})$ in the assumption for the minimal cover problem is created by a Henneberg sequence, it has a set of independent edges. Since $\mathbb{G}$ is a subgraph of $\overline{\mathbb{G}}$, it also has a set of independent edges. In merging, since each pre-merged rigid subgraph is created by a Henneberg sequence, $\mathbb{G}$ has a set of independent edges.

The minimal cover problem can be posed using concepts from lattice theory. Let $\mathcal{G}$ denote the set of all simple graphs ${ }^{3} \mathbb{G}=(\mathcal{V}, \mathcal{L})$ with vertex set $\mathcal{V}$ including the graph with empty edge set which is called the edgeless graph. Containment, denoted by $\subset$, is a relation on $\mathcal{G}$ such that $\mathbb{G}_{1}=\left(\mathcal{V}, \mathcal{L}_{1}\right)$ is contained in $\mathbb{G}_{2}=\left(\mathcal{V}, \mathcal{L}_{2}\right)$ if $\mathcal{L}_{1} \subset \mathcal{L}_{2}$. Containment is a partial ordering on $\mathcal{G}$, and the complete graph and edgeless graph are $\mathcal{G}$ 's largest and smallest elements with respect to this ordering (see for example Birkhoff [5] and MacLane and Birkhoff [19]). Every graph $\mathbb{G}$ in $\mathcal{G}$ is contained in at least one rigid graph. We are interested in minimally rigid graphs in $\mathcal{G}$ that contain $\mathbb{G}$, and we call such a minimally rigid graph a minimal cover. The set of edges we want to preserve is represented by $\mathcal{L}$.

We can define the minimal cover problem in terms of partial ordering and graph rigidity. Suppose that a graph $\mathbb{G}=(\mathcal{V}, \mathcal{L}) \in \mathcal{G}$, which satisfies the assumption of the minimal cover problem, is given. The minimal cover problem is to find some $\mathbb{G}^{*}=\left(\mathcal{V}, \mathcal{L}^{*}\right) \in \mathcal{G}$ such that $\mathbb{G} \subset \mathbb{G}^{*}$ and $\mathbb{G}^{*}$ is minimally rigid. In other words, we want to find a set of new edges, namely $\mathcal{L}_{\text {new }}$, between the vertices of $\mathcal{V}$ to add to the set $\mathcal{L}$ such that the resulting graph $\mathbb{G}^{*}=\left(\mathcal{V}, \mathcal{L}^{*}\right)$ is minimally rigid, where $\mathcal{L}^{*}=\mathcal{L} \cup \mathcal{L}_{\text {new }}$. We note that $\mathbb{G}^{*}$ is not necessarily unique.

In splitting, for example, all the links that need not be removed are preserved in each post-split sub-formation, and a minimum number of links is inserted into each post-split sub-formation to regain rigidity in each non-rigid post-split sub-formation. In merging, all the present links are maintained and a minimum number of new links is inserted between sub-formations to result in a single rigid formation.

Recall that generic rigidity is directly related to the rank of a matrix. As such, it has all the "exchange properties" associated with the independence of rows of a

\footnotetext{
${ }^{3}$ By definition a graph contains neither a "loop" nor "multiple edges." A loop is an edge joining a vertex to itself. Multiple edges are several edges joining the same two vertices. In a multigraph, both loops and multiple edges are allowed. When there is any danger of confusion, graphs are called simple graphs.
} 
matrix [27]. For example, we note that minimally rigid graphs are also maximally independent graphs, corresponding to bases in vector spaces as minimal spanning sets and maximal independent sets. Given any independent set of edges $\mathcal{I}$ which is a subset of a basis (maximally independent set of edges) $\mathcal{B}$ for a vertex set $\mathcal{V}$, a set of edges $\mathcal{J}$ is a minimal cover of $\mathcal{I}$, if the union of $\mathcal{I}$ and $\mathcal{J}$ is a (new) basis $\mathcal{B}^{\prime}$ for $\mathcal{V}$. Moreover, given an independent set of edges and some spanning set containing this set (e.g., the complete graph), we can extend the the independent set to a basis in a "greedy" manner, i.e., the new edges are added to the independent set one step at a time.

3.1. Planar Case. One crude approach to solve the minimal cover problem in 2-dimensional space is based on the "generate and test" method. It is as follows: Given a graph $\mathbb{G}=(\mathcal{V}, \mathcal{L}) \in \mathcal{G}$, we test whether with the addition of a new edge $e$, the graph $\mathbb{G}=(\mathcal{V}, \mathcal{L} \cup\{e\})$ is independent or not. If it is then we add $e$ to $\mathcal{L}$. We repeat, adding such new edges, until we have a set of $2 n-3$ independent edges where $n$ is the number of vertices. The resulting graph with the vertex set $\mathcal{V}$ and those $2 n-3$ independent edges is the minimal cover of $\mathbb{G}$. This approach works based on random trials. If a new randomly generated edge gives us a set of independent edges then it turns out to be a success, otherwise it is a failure.

Now we present a systematic strategy to solve the minimal covering problem. First, we state the following lemma, then we introduce two types of reduction steps that will be used in the sequel.

Lemma 13. Let $\mathbb{G}=(\mathcal{V}, \mathcal{L})$ denote a graph satisfying the assumption of the minimal cover problem in 2-dimensional space. Then there exists a vertex of degree 3 or less in $\mathbb{G}$.

Proof. From the definition of a set of independent edges in $\S 2.3,|\mathcal{L}| \leq 2|\mathcal{V}|-3$ which implies $2|\mathcal{L}| \leq 4|\mathcal{V}|-6$. Hence there exists a vertex of degree 3 or less in $\mathbb{G}=(\mathcal{V}, \mathcal{L})$.

Reduction Step - Type I: Let $\mathbb{G}=(\mathcal{V}, \mathcal{L})$ be a graph satisfying the assumption of the minimal cover problem in 2-dimensional space. Let $i$ be a vertex of degree $\rho(i)$, where $\rho(i) \in\{0,1,2\}$. Suppose that we create a set of $2-\rho(i)$ new edges incident to $i$ and denote this set of new edges by $\mathcal{L}_{i_{\text {new }}}$. Let $\mathbb{G}^{\prime}=\left(\mathcal{V}, \mathcal{L}^{\prime}\right)$ denote this new graph where $\mathcal{L}^{\prime}=\mathcal{L} \cup \mathcal{L}_{i_{\text {new }}}$. We register $\mathcal{L}_{i_{\text {new }}}$ to use in subsequent steps. It can be easily verified that the set of edges $\mathcal{L}^{\prime}$ is independent. Now let us remove the vertex $i$ and all the edges incident to $i$ in $\mathbb{G}^{\prime}=\left(\mathcal{V}, \mathcal{L}^{\prime}\right)$. Let $\mathbb{G}^{\prime \prime}=\left(\mathcal{V}^{\prime}, \mathcal{L}^{\prime \prime}\right)$ denote this reduced graph where $\mathcal{V}^{\prime}=\mathcal{V} \backslash\{i\}$ and $\mathcal{L}^{\prime \prime}=\mathcal{L}^{\prime} \backslash\{$ all edges incident to $i\}$. It can again be verified that the edge set of $\mathbb{G}^{\prime \prime}=\left(\mathcal{V}^{\prime}, \mathcal{L}^{\prime \prime}\right)$ is also independent. Therefore there exists a vertex of degree 3 or less in $\mathbb{G}^{\prime \prime}$ by Lemma 13 .

Reduction Step - Type II: Let $\mathbb{G}=(\mathcal{V}, \mathcal{L})$ be a graph satisfying the assumption of the minimal cover problem in 2-dimensional space. Let $i$ be a vertex of degree 3 
and adjacent to a set of vertices denoted by $\mathcal{N}_{i}$. We remove $i$ and its three edges and create a new edge (precisely one edge) between arbitrary vertices in $\mathcal{N}_{i}$ forming a reduced graph $\mathbb{G}^{\prime}=\left(\mathcal{V}^{\prime}, \mathcal{L}^{\prime}\right)$ where $\mathcal{V}^{\prime}=\mathcal{V} \backslash\{i\}$ and $\mathcal{L}^{\prime}=\mathcal{L} \backslash\{$ all edges incident to $i\} \cup\left\{\right.$ a new edge between the vertices in $\left.\mathcal{N}_{i}\right\}$ such that $\mathcal{L}^{\prime}$ is a set of independent edges. ${ }^{4}$ Therefore there exists a vertex of degree 3 or less in $\mathbb{G}^{\prime}$ by Lemma 13 .

Now we present a reduction sequence in which the two types of reduction steps previously described are used as main steps. This sequence is used to reduce a set of independent edges down to a set of two vertices connected by an edge.

Reduction Sequence: Suppose that a graph $\mathbb{G}=(\mathcal{V}, \mathcal{L})$ satisfying the assumption of the minimal cover problem in 2-dimensional space is given. From Lemma 13 it follows that there exists a vertex of degree 3 or less in $\mathbb{G}$. Hence, at least one of the reduction steps (type I or type II) can be applied to $\mathbb{G}$. Let us note that the reduced graphs we obtain after applying any one of these reduction steps are also independent. We apply these two types of steps repeatedly until we are left with only two vertices. While we apply the reduction steps to the vertices, we number those vertices in a descending order as $n, n-1, n-2, \ldots, 4,3$. For example, we number the vertex removed in the first reduction step with order $n$, we number the vertex removed at the second reduction step with $n-1$ and so on until we are left with two vertices. The last vertex on which a reduction step is applied is numbered with 3 . Then the very last two remaining vertices are numbered with orders 2 and 1 . Each time we apply the reduction step type I on a vertex $i$, we keep registering its new set of edges $\mathcal{L}_{i_{\text {new }}}$ as described in reduction step type I. Depending on the initial set of independent edges, there may or may not be left an edge between the last two vertices after the execution of the reduction sequence. If there is no edge between them at the end of the reduction sequence, we create such an edge and register it as $\mathcal{L}_{2_{\text {new }}}$. If there is already an edge between ordered vertices 1 and 2 , then we register $\mathcal{L}_{2_{\text {new }}}=\emptyset$. The union of the registered sets of the new edges is $\mathcal{L}_{\text {new }}=\bigcup_{i} \mathcal{L}_{i_{\text {new }}}$ where $i$ denotes the label of the vertices removed with a type I reduction step and the vertex with order 2 .

Theorem 14. (Planar Minimal Cover Theorem) Let $\mathbb{G}=(\mathcal{V}, \mathcal{L})$ be a graph satisfying the assumption of the minimal cover problem in 2-dimensional space. Suppose that we apply the reduction sequence described above on $\mathbb{G}$ and find $\mathcal{L}_{\text {new }}$. Then $\mathbb{G}^{*}=\left(\mathcal{V}, \mathcal{L} \cup \mathcal{L}_{\text {new }}\right)$ is a minimal cover of $\mathbb{G}$.

Proof. To prove that $\mathbb{G}^{*}$ is a minimal cover of $\mathbb{G}$, we need to prove that $\mathbb{G} \subset \mathbb{G}^{*}$ and $\mathbb{G}^{*}$ is minimally rigid. First, it is easy to verify that $\mathbb{G}=(\mathcal{V}, \mathcal{L}) \subset \mathbb{G}^{*}=\left(\mathcal{V}, \mathcal{L} \cup \mathcal{L}_{\text {new }}\right)$. Second, starting from the vertices 1 and 2 and the edge $(1,2)$, let us suppose that we apply the reduction sequence in reverse order including the edges in $\mathcal{L}_{\text {new }}$. Note that

\footnotetext{
${ }^{4}$ The existence of such a new edge can be seen from the proof of the edge splitting operation in Whiteley [27].
} 
the reduction step types I and II applied in the reverse sequence are nothing but the vertex addition and edge splitting operations in a Henneberg type sequence. Hence the reduction sequence applied in reverse order is actually a Henneberg sequence. At the end of such a Henneberg sequence, we obtain $\mathbb{G}^{*}=\left(\mathcal{V}, \mathcal{L} \cup \mathcal{L}_{\text {new }}\right)$, and it is minimally rigid (From $\S 2.4$, the graphs generated by a Henneberg sequence are minimally rigid.).

Note that $\mathcal{L}_{\text {new }}$ obtained in the reduction sequence is not unique because the edges in $\mathcal{L}_{\text {new }}$ depend on the choice of the order of vertices in the reduction sequence. Therefore a minimal cover of a set of independent edges is not unique.

We will not consider algorithmic complexities in this paper. But one can argue that each time the reduction step type II is applied, there are up to three possible insertions for a new edge. This results in an exponential algorithm for the reduction sequence. To overcome this problem, the approach for the spatial case presented in the sequel can be easily applied to the planar case. Alternatively one can use a polynomial time algorithm called "the pebble game" for the planar case. There is a recent paper by Berg and Jordan [4] which addresses this kind of algorithms.

3.2. Spatial Case. The approach for the minimal cover problem based on "generate and test" cannot be applied in 3-dimensional space simply because of the nonavailability of an appropriate analog of Laman's Theorem in 3-dimensional space.

The approach in the Planar Minimal Cover Theorem can be translated to 3dimensional space with an additional condition in the order of the reduction sequence. The reason behind this condition is that if a reduction sequence similar to the planar case is applied then there is a possibility of reaching a graph with a set of vertices all of which are of degree 5 . If the conjectures for adding a 5 -valent vertex to minimally rigid graphs that we had explained in $\S 2.4$ are proven, then the reduction sequence presented for the planar case can be easily extended to 3-dimensional space. Since this is a long-standing unsolved problem, to get around those difficulties, we present an alternative reduction sequence with an additional condition in the ordering of reduction sequence. We have the following lemma in 3-dimensional space.

Lemma 15. Let $\mathbb{G}=(\mathcal{V}, \mathcal{L})$ denote a graph satisfying the assumption of the minimal cover problem in 3-dimensional space. Then there exists a vertex of $\mathbb{G}$ of degree 4 or less.

Proof. In merging, by the assumption, each pre-merged subgraph is created by a Henneberg sequence. Therefore there exits a vertex of degree 4 or less in each subgraph.

In splitting, by the assumption, recall that each post-split subgraph denoted by $\mathbb{G}=(\mathcal{V}, \mathcal{L})$ is a subset of a bigger graph $\overline{\mathbb{G}}=(\overline{\mathcal{V}}, \overline{\mathcal{L}})$ and $\overline{\mathbb{G}}$ is a minimally rigid graph created by the vertex addition and edge splitting operations in 3-dimensional space. Let the vertices in $\overline{\mathcal{V}}$ be indexed $\{1,2, \ldots, n\}$ with respect to their order of addition 
in the Henneberg sequence. We will prove that the vertex with the highest index in each post-split subgraph cannot be of degree 5 or higher. Let us denote the highest index in each subgraph $\mathbb{G}$ by a generic label $k$.

First let us suppose that vertex $k$ was created, as part of the Henneberg sequence construction of $\overline{\mathbb{G}}$, by the vertex addition operation, through inserting the edges $(k, i)$, $(k, j),(k, l)(i, j, l<k)$. All the vertices with a higher index created by the vertex addition operation connected to this vertex are not in this subgraph $\mathbb{G}$ or $k$ is the maximum index in $\overline{\mathbb{G}}$. Hence the edges between higher indexed vertices (e.g. $q>k$ ) and $k$ are not in the subgraph. All the vertices with a higher index created by edge splitting one of the edges $(k, i),(k, j),(k, l)$, do not imply an increase of the degree of $k$, considered as a vertex of the subgraph $\mathbb{G}$, and furthermore these associated edges are not in the same subgraph, because they are either removed in splitting operation or $k$ is the maximum index in $\overline{\mathbb{G}}$. Hence the degree of vertex $k$ in the subgraph can be at most 3 . It is less than 3 if at least one of $i, j, l$ is not in $\mathbb{G}=(\mathcal{V}, \mathcal{L})$.

Second, let us suppose that the vertex was created by the edge splitting operation: splitting the edge $e_{k}$, linking two of the vertices $i, j, l, m$, and creating the edges $(k, i),(k, j),(k, l),(k, m)(i, j, l, m<k)$. All the vertices with a higher index created by the vertex addition operation connected to the vertex $k$ are not in the subgraph $\mathbb{G}$. Hence the edges connecting them to $k$ are not in the subgraph $\mathbb{G}$. If a vertex $q$ with a higher index $(q>k)$ is created by an edge splitting operation by splitting one of the edges incident to $k$ (i.e., $(k, i),(k, j),(k, l),(k, m))$ in the Henneberg sequence, then this vertex $q>k$ would not be in the remaining subgraph, and the degree of the vertex $k$ would be of degree 3 or less. Therefore the degree of vertex $k$ can be at most 4. If any of the vertices $i, j, l, m$ are not in $\mathbb{G}(\mathcal{V}, \mathcal{L})$, the degree of $k$ will be less than 4. If the degree is 4 , it has the edges $(k, i),(k, j),(k, l),(k, m)$.

The two reduction steps presented for the planar case can be directly extended for 3-dimensional space.

Reduction Step - Type I: Let $\mathbb{G}=(\mathcal{V}, \mathcal{L})$ be a graph satisfying the assumption of the minimal cover problem in 3-dimensional space. Let $i$ be a vertex of degree $\rho(i)$, where $\rho(i) \in\{0,1,2,3\}$. Suppose that we create a set of $3-\rho(i)$ new edges incident to $i$ and denote this set of new edges by $\mathcal{L}_{i_{\text {new }}}$. Let $\mathbb{G}^{\prime}=\left(\mathcal{V}, \mathcal{L}^{\prime}\right)$ denote this new graph where $\mathcal{L}^{\prime}=\mathcal{L} \cup \mathcal{L}_{i_{\text {new }}}$. We register $\mathcal{L}_{i_{\text {new }}}$ to use in subsequent steps. It can be easily verified that the set of edges $\mathcal{L}^{\prime}$ is independent. Now let us remove the vertex $i$ and all the edges incident to $i$ in $\mathbb{G}^{\prime}=\left(\mathcal{V}, \mathcal{L}^{\prime}\right)$. Let $\mathbb{G}^{\prime \prime}=\left(\mathcal{V}^{\prime}, \mathcal{L}^{\prime \prime}\right)$ denote this reduced graph where $\mathcal{V}^{\prime}=\mathcal{V} \backslash\{i\}$ and $\mathcal{L}^{\prime \prime}=\mathcal{L}^{\prime} \backslash\{$ all edges incident to $i\}$. It can be easily verified that $\mathbb{G}^{\prime \prime}=\left(\mathcal{V}^{\prime}, \mathcal{L}^{\prime \prime}\right)$ also satisfies the assumption of the minimal cover problem. Therefore there exists a vertex of degree 4 or less in $\mathbb{G}^{\prime \prime}$ by Lemma 15 .

Reduction Step - Type II: Let $\mathbb{G}=(\mathcal{V}, \mathcal{L})$ be a graph satisfying the assumption of the minimal cover problem in 3-dimensional space. Let $i$ be a vertex of degree 4 and adjacent to a set of vertices denoted by $\mathcal{N}_{i}$. We remove $i$ and its four edges and 
create a new edge (precisely one edge) between arbitrary vertices in $\mathcal{N}_{i}$ forming a reduced graph $\mathbb{G}^{\prime}=\left(\mathcal{V}^{\prime}, \mathcal{L}^{\prime}\right)$ where $\mathcal{V}^{\prime}=\mathcal{V} \backslash\{i\}$ and $\mathcal{L}^{\prime}=\mathcal{L} \backslash\{$ all edges incident to $i\} \cup\left\{\right.$ a new edge between the vertices in $\left.\mathcal{N}_{i}\right\}$ such that $\mathbb{G}^{\prime}$ satisfies the assumption of the minimal cover problem. ${ }^{5}$ Therefore there exists a vertex of degree 4 or less in $\mathbb{G}^{\prime}$ by Lemma 15 .

Now we present the modified reduction sequence, in which the two types of reduction steps previously described are used as main steps. This sequence is used to reduce a graph satisfying the assumption of the minimal cover problem in 3-dimensional space down to a set of three vertices. It makes use of the order of vertices in the Henneberg sequence that was used to create the original graph $\overline{\mathbb{G}}=(\overline{\mathcal{V}}, \overline{\mathcal{L}})$.

Reduction Sequence: Let $\overline{\mathbb{G}}=(\overline{\mathcal{V}}, \overline{\mathcal{L}})$ be a minimally rigid graph created by the vertex addition and edge splitting operations in 3-dimensional space, where vertices are indexed $\{1,2, \ldots, n\}$ with respect to their order of addition in the Henneberg sequence. If $i$ denotes a vertex added by the edge splitting operation, then let $e_{i}$ denote the edge removed in this operation. Let the graph $\mathbb{G}=(\mathcal{V}, \mathcal{L})$ be created by removing some of the edges and vertices of the graph $\overline{\mathbb{G}}=(\overline{\mathcal{V}}, \overline{\mathcal{L}})$. To complete $\mathbb{G}=(\mathcal{V}, \mathcal{L})$ to a minimally rigid graph, we do the following. Starting from the vertex with the highest index, we apply reductions steps type I and II repeatedly on the vertices with descending order of indices. For example, let $i$ denote the vertex with the highest index in the remaining graph at some step in the reduction sequence. If $i$ is of degree $\rho(i)$, where $\rho(i) \leq 3$, we apply reduction step type I. Each time we apply the reduction step type I on a vertex $i$, we keep registering its new set of edges $\mathcal{L}_{i_{\text {new }}}$ as described in reduction step type I. If the vertex $i$ is of degree 4, we then apply reduction step type II by inserting the edge $e_{i}$. We continue this until three vertices are left. Depending on the initial set of independent edges, there may or may not be three edges left between the last three vertices after the execution of the reduction sequence. If there are not three edges between them at the end of the reduction sequence, we complete the number of edges to three and register them as $\mathcal{L}_{3_{\text {new }}}$. If there are already three edges between vertices labelled 1,2 and 3, then we register $\mathcal{L}_{3_{\text {new }}}=\emptyset$. The union of the registered sets of the new edges is $\mathcal{L}_{\text {new }}=\bigcup_{i} \mathcal{L}_{i_{\text {new }}}$ where $i$ denotes the label of the vertices removed with a type I reduction step and the vertex with index 3 .

Lemma 16. At each reduction step, the vertex with the highest index is of degree 4 or less.

Proof. The proof is the same as the proof of splitting part of Lemma 15.

Theorem 17. (Spatial Minimal Cover Theorem) Let $\mathbb{G}=(\mathcal{V}, \mathcal{L})$ be a graph satisfying the assumption of the minimal cover problem in 3-dimensional space. Sup-

\footnotetext{
${ }^{5}$ The existence of such an edge will become clear in the special reduction sequence presented in the sequel.
} 
pose that we apply the reduction sequence described above on $\mathbb{G}$ and find $\mathcal{L}_{\text {new }}$. Then $\mathbb{G}^{*}=\left(\mathcal{V}, \mathcal{L} \cup \mathcal{L}_{\text {new }}\right)$ is a minimal cover of $\mathbb{G}$.

Proof. To prove that $\mathbb{G}^{*}$ is a minimal cover of $\mathbb{G}$, we need to prove that $\mathbb{G} \subset \mathbb{G}^{*}$ and $\mathbb{G}^{*}$ is minimally rigid. First, it is easy to see that $\mathbb{G}=(\mathcal{V}, \mathcal{L}) \subset \mathbb{G}^{*}=\left(\mathcal{V}, \mathcal{L} \cup \mathcal{L}_{\text {new }}\right)$. Second, starting from the vertices 1,2 and 3 and the edges $(1,2),(1,3),(2,3)$ let us suppose that we apply the reduction sequence in reverse order including the edges in $\mathcal{L}_{\text {new }}$. Note that the reduction step types I and II applied in the reverse order are nothing but the vertex addition and edge splitting operations used in a Henneberg type sequence. Hence the reduction sequence in reverse order is actually a Henneberg sequence. At the end of such a Henneberg sequence, we obtain $\mathbb{G}^{*}=\left(\mathcal{V}, \mathcal{L} \cup \mathcal{L}_{\text {new }}\right)$, and it is minimally rigid (From $\S 2.4$, the graphs generated by a Henneberg sequence are minimally rigid.).

As in the planar case, $\mathcal{L}_{\text {new }}$ obtained in the reduction sequence is not unique because the edges in $\mathcal{L}_{\text {new }}$ depend on the choice in the reduction sequence. Therefore a minimal cover of a set of independent edges is not unique.

We note that the reduction strategies developed in this paper can be extended to include other types of operations such as vertex splitting.

4. Splitting Formations. To find a strategy for splitting a rigid formation into two rigid post-split sub-formations, it is convenient to introduce a suitable definition of the splitting problem in terms of graph rigidity. Let $\mathbb{G}=(\mathcal{V}, \mathcal{L})$ be a minimally rigid graph. Let $\mathcal{V}_{1}$ and $\mathcal{V}_{2}$ represent the two subsets of $\mathcal{V}$ such that $\mathcal{V}_{1} \cup \mathcal{V}_{2}=\mathcal{V}$ and $\mathcal{V}_{1} \cap \mathcal{V}_{2}=\emptyset$. Let $\mathcal{L}_{1} \subset \mathcal{L}$ be the set of all edges whose both end-vertices are in $\mathcal{V}_{1}$ and $\mathcal{L}_{2} \subset \mathcal{L}$ be the set of all edges whose both end-vertices are in $\mathcal{V}_{2}$. Let $\mathcal{L}_{r}=\mathcal{L} \backslash\left(\mathcal{L}_{1} \cup \mathcal{L}_{2}\right)$ be the set of all edges whose one end-vertex is in $\mathcal{L}_{1}$ and the other end-vertex is in $\mathcal{L}_{2}$. Let $\mathbb{G}_{1}=\left(\mathcal{V}_{1}, \mathcal{L}_{1}\right)$ and $\mathbb{G}_{2}=\left(\mathcal{V}_{2}, \mathcal{L}_{2}\right)$. When the graph $\mathbb{G}=(\mathcal{V}, \mathcal{L})$ is split into $\mathbb{G}_{1}=\left(\mathcal{V}_{1}, \mathcal{L}_{1}\right)$ and $\mathbb{G}_{2}=\left(\mathcal{V}_{2}, \mathcal{L}_{2}\right)$, all edges in $\mathcal{L}_{r}$ are removed. The splitting problem is to find new sets of edges $\mathcal{L}_{1_{\text {new }}}$ to insert into $\mathbb{G}_{1}$ and $\mathcal{L}_{2_{\text {new }}}$ to insert into $\mathbb{G}_{2}$ such that the resulting graphs $\mathbb{G}_{1}^{*}=\left(\mathcal{V}_{1}, \mathcal{L}_{1} \cup \mathcal{L}_{1_{\text {new }}}\right)$ and $\mathbb{G}_{2}^{*}=\left(\mathcal{V}_{2}, \mathcal{L}_{2} \cup \mathcal{L}_{2_{\text {new }}}\right)$ are minimally rigid.

With the minimal cover problem in mind as defined in the previous section, the splitting problem reduces to finding the minimal cover of $\mathbb{G}_{1}=\left(\mathcal{V}_{1}, \mathcal{L}_{1}\right)$ and $\mathbb{G}_{2}=$ $\left(\mathcal{V}_{2}, \mathcal{L}_{2}\right)$. As detailed in the analysis in the previous section, the underlying graphs of the resulting post-split sub-formations determined by the reduction sequences are minimally rigid by Theorem 14 and Theorem 17 .

5. Merging Formations. As we did in the case of the splitting problem, we introduce a suitable definition of the merging problem in terms of graph rigidity.

Let $\mathbb{G}_{1}=\left(\mathcal{V}_{1}, \mathcal{L}_{1}\right)$ and $\mathbb{G}_{2}=\left(\mathcal{V}_{2}, \mathcal{L}_{2}\right)$ be two minimally rigid graphs representing the underlying graphs of two minimally rigid point formations. The merging problem is to find a new set of edges $\mathcal{L}_{\text {new }}$ to insert between $\mathbb{G}_{1}$ and $\mathbb{G}_{2}$ by choosing one 
end-vertex in $\mathcal{V}_{1}$ and the other end-vertex in $\mathcal{V}_{2}$ such that the resulting graph $\mathbb{G}^{*}=$ $\left(\mathcal{V}_{1} \cup \mathcal{V}_{2}, \mathcal{L}_{1} \cup \mathcal{L}_{2} \cup \mathcal{L}_{\text {new }}\right)$ is minimally rigid.

As in the case of splitting, the merging problem reduces to finding the minimal cover of $\mathbb{G}^{\prime}=\left(\mathcal{V}_{1} \cup \mathcal{V}_{2}, \mathcal{L}_{1} \cup \mathcal{L}_{2}\right)$. We exemplify this in the sequel.

Example: Let $\mathbb{G}_{1}=\left(\mathcal{V}_{1}, \mathcal{L}_{1}\right)$ and $\mathbb{G}_{2}=\left(\mathcal{V}_{2}, \mathcal{L}_{2}\right)$ be two minimally rigid graphs in 2-dimensional space. Suppose that we apply the reduction sequence first on $\mathbb{G}_{2}=$ $\left(\mathcal{V}_{2}, \mathcal{L}_{2}\right)$ as described in $\S 3.1$. Since $\mathbb{G}_{2}$ is minimally rigid, we obtain two vertices connected by an edge at the end of the reduction sequence on $\mathbb{G}_{2}$ without inserting any new edges. Let us denote this remaining edge by $(i, j)$. At this intermediate step, we are left with $\mathbb{G}_{1}=\left(\mathcal{V}_{1}, \mathcal{L}_{1}\right)$ and the edge $(i, j)$ on which we continue applying the reduction sequence. Suppose that we apply the reduction step type I on the vertex $i$, by inserting an edge $(i, k)$ where $k \in \mathcal{V}_{1}$. Then we apply the reduction step type I on the vertex $j$ by inserting the edges $(j, k),(j, l)$ or by inserting the edges $(j, l),(j, r)$ where $k, l, r \in \mathcal{V}_{1}$. After applying those reduction steps on vertices $i, j$, we are left with the graph $\mathbb{G}_{1}=\left(\mathcal{V}_{1}, \mathcal{L}_{1}\right)$ only, and we apply the reduction sequence on $\mathbb{G}_{1}$ without inserting any new edges. Therefore the two possible combinations of merging $\mathbb{G}_{1}$ and $\mathbb{G}_{2}$ create the set of edges $(i, k),(j, k),(j, l)$ or $(i, k),(j, l),(j, r)$. We depict these two different strategies of merging two rigid sub-formations in Figures $11 \mathrm{a}$ and b. $\mathbb{G}_{1}$ and $\mathbb{G}_{2}$ denote the underlying graphs of these two rigid point formations.

We can pursue a different strategy. Let $\mathbb{G}_{1}=\left(\mathcal{V}_{1}, \mathcal{L}_{1}\right)$ and $\mathbb{G}_{2}=\left(\mathcal{V}_{2}, \mathcal{L}_{2}\right)$ be two minimally rigid graphs in 2-dimensional space. Suppose that we apply the reduction sequence first on $\mathbb{G}_{2}=\left(\mathcal{V}_{2}, \mathcal{L}_{2}\right)$ as described in $\S 3.1$ until we are left with three vertices $i, j, k$ connected by three edges $(i, j),(i, k),(j, k)$. Since $\mathbb{G}_{2}$ is minimally rigid, we obtain these three vertices connected by three edges at the end of the reduction sequence on $\mathbb{G}_{2}$ without inserting any new edges. First, let us insert a new edge $(i, r)$ where $r \in \mathcal{V}_{1}$. Now the vertex $i$ is of degree 3 . Then, we can apply the reduction step type II on the vertex $i$ by inserting the edge $(j, r)$. Note that while $(i, r)$ is a new inserted edge which needs to be registered in the reduction sequence process, $(j, r)$ is not. $(j, r)$ is simply a result of reduction step type II. Now let us insert a new edge $(j, s)$ to make the vertex $j$ of degree 3 where $s \in \mathcal{V}_{1}$. Then we can apply the reduction step type II on the vertex $j$ by inserting an edge $(k, s)$. Note again that $(j, s)$ is a new edge which needs to be registered in the reduction sequence process but $(k, s)$ is not. Then we apply the reduction step type I on the vertex $k$ by inserting a new edge $(k, t)$ where $t \in \mathcal{V}_{1}$. At this stage, we are only left with $\mathbb{G}_{1}$. Then we continue the reduction sequence on $\mathbb{G}_{1}$. Since $\mathbb{G}_{1}$ is minimally rigid, the reduction sequence can be applied without inserting any other extra edges. Therefore another strategy for merging $\mathbb{G}_{1}$ and $\mathbb{G}_{2}$ creates the set of edges $(i, r),(j, s),(k, t)$. We depict this strategy in Figure 11c.

Merging rigid bodies in 3-dimensional space has been studied in rigidity theory. We refer the reader to Whiteley [27] for a detailed explanation. Here, we use the 
solution of the minimal cover problem to determine these new links. It can be verified that six links are needed in 3-dimensional space to merge two minimally rigid subformations to form a minimally rigid post-merged formation provided that we use at least three points in each sub-formation as an end-point of these six new links. Here, we depict three possible strategies to determine these six links (Figures 12a, b, c) and explain one of them in detail (Figure 12a). One can find different strategies by using a modified version of the idea presented here by selecting a different combination of vertices and reduction steps type I and II. Here, we do not go into each such combinations because of space constraints and because the idea is essentially similar to the planar version.

Let $\mathbb{G}_{1}=\left(\mathcal{V}_{1}, \mathcal{L}_{1}\right)$ and $\mathbb{G}_{2}=\left(\mathcal{V}_{2}, \mathcal{L}_{2}\right)$ be two minimally rigid graphs in 3dimensional space. Suppose that we apply the reduction sequence first on $\mathbb{G}_{2}=$ $\left(\mathcal{V}_{2}, \mathcal{L}_{2}\right)$ as described in $\S 3.1$. Since $\mathbb{G}_{2}$ is minimally rigid, we obtain three vertices connected by three edges at the end of the reduction sequence on $\mathbb{G}_{2}$ without inserting any new edges. Let us denote this remaining vertices by $i, j, k$ and edges by $(i, j),(i, k),(j, k)$. At this intermediate step, we are left with $\mathbb{G}_{1}=\left(\mathcal{V}_{1}, \mathcal{L}_{1}\right)$ and the edge $(i, j),(i, k),(j, k)$ on which we continue applying the reduction sequence. Suppose that we apply the reduction step type I on the vertex $i$, by inserting an edge $(i, l)$ where $l \in \mathcal{V}_{1}$. Then we apply the reduction step type I on the vertex $j$ by inserting the edges $(j, r),(j, s)$ where $r, s \in \mathcal{V}_{1}$. Then we apply the reduction step type I on the vertex $k$ by inserting the edges $(k, t),(k, u),(k, v)$ where $t, u, v \in \mathcal{V}_{1}$. After applying those reduction steps on vertices $i, j, k$, we are left with the graph $\mathbb{G}_{1}=\left(\mathcal{V}_{1}, \mathcal{L}_{1}\right)$ only, and we apply the reduction sequence on $\mathbb{G}_{1}$ without inserting any new edges. Therefore the two possible combinations of merging $\mathbb{G}_{1}$ and $\mathbb{G}_{2}$ create the set of edges $(i, l),(j, r),(j, s),(k, t),(k, u),(k, v)$. We depict this strategy of merging two rigid sub-formations in Figure 5.12(a). $\mathbb{G}_{1}$ and $\mathbb{G}_{2}$ denote the underlying graphs of these two rigid point formations.

6. The Closing Ranks Problem. The closing ranks problem can be formulated as follows. We begin with a minimally rigid graph $\mathbb{G}=(\mathcal{V}, \mathcal{L})$, which is the underlying graph of a minimally rigid point formation in real $d$-dimensional space $\{d$ $=2$ or 3$\}$. Let $i$ be a vertex in $\mathcal{V}$ with a neighbor set $\mathcal{N}_{i}$. Let $\mathcal{L}_{i}$ denote the set of incident edges to $i$. Suppose that $i$ and $\mathcal{L}_{i}$ are removed from $\mathbb{G}$. Let $\mathbb{G}^{*}\left(\mathcal{V}^{*}, \mathcal{L}^{*}\right)$ denote the remaining graph (i.e., $\mathcal{V}^{*}=\mathcal{V} \backslash\{\mathrm{i}\}$ and $\mathcal{L}^{*}=\mathcal{L} \backslash \mathcal{L}_{i}$ ). The closing ranks problem is to find the set of new edges $\mathcal{L}_{\text {new }}$ between the vertices in $\mathcal{N}_{i}$ so that the resulting graph $\left(\mathcal{V}^{*}, \mathcal{L}^{*} \cup \mathcal{L}_{\text {new }}\right)$ is minimally rigid.

We have the following theorem:

THEOREM 18. Assume that a vertex of degree $k$ and its incident edges are removed from a minimally rigid graph in $d$-dimensional space. To regain minimal rigidity, $k-d$ new edges (or no edges if $k=d$ ) should be inserted and those new links can be inserted 


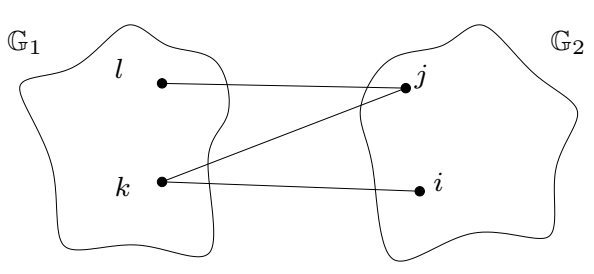

(a)

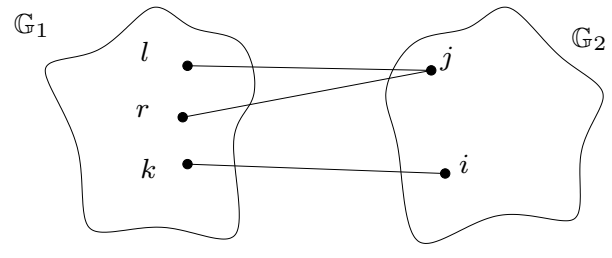

(b)

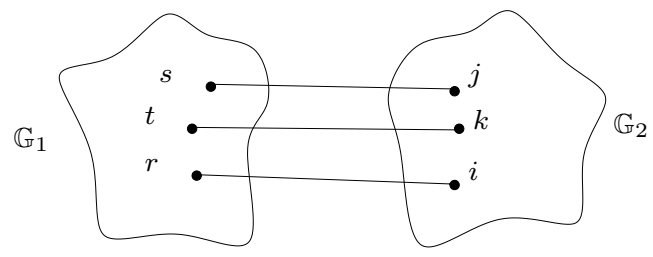

(c)

FIG. 11. Merging rigid sub-formations in 2-dimensional space.

between only the neighbors of the removed vertex, without using any other vertex as an end-vertex of those inserted edges.

In the sequel, we give the proofs for removing vertices of any degree both in 2- and 3 -dimensional space. When a vertex of degree $k$ and its incident edges are removed from a minimally rigid graph, $k-d$ new edges should be inserted to regain rigidity.

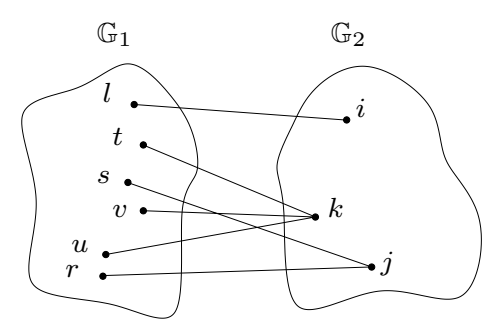

(a)

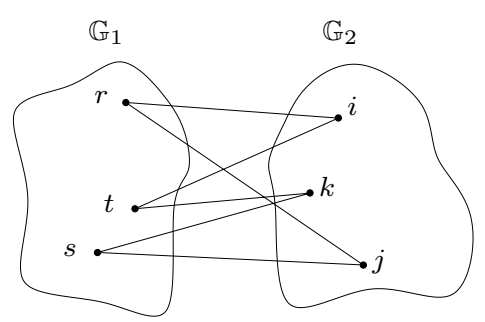

(b)

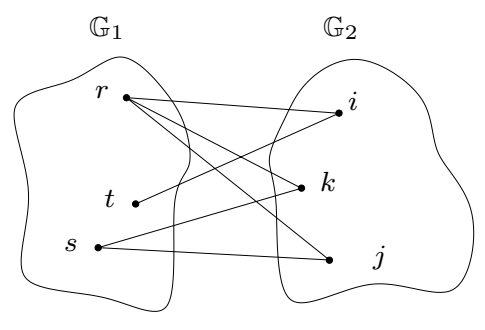

(c)

FIG. 12. Three different strategies of merging rigid sub-formations in 3-dimensional space. 
This can be seen easily from the count of vertices and edges in minimally rigid graphs. In the sequel, we show that it is indeed possible to insert those new links only between the neighbors of the removed vertex and regain minimal rigidity, without using any other vertex as an end-vertex of those inserted edges. For finding those edges, one can simply insert possible combinations of new edges and test minimal rigidity. The number of possible combinations of insertions in the case of the removal of a vertex of degree $k$ in a minimally rigid graph in $d$-space is

$$
\left(\begin{array}{c}
\left(\begin{array}{c}
k \\
2
\end{array}\right) \\
k-d
\end{array}\right)=\frac{k(k-1)}{2(k-d)}
$$

"Generate and test" type strategy is efficient to try this number of combinations. Alternatively, one might use a procedure similar to the one that is used in the minimal cover problem. Here, our main concern is the existence of new edges between the neighbors of the removed vertex such that insertion of those edges results in minimal rigidity.

6.1. Planar Case. Removing a vertex with two edges: A minimally rigid graph $\mathbb{G}=(\mathcal{V}, \mathcal{L})$ is shown in Figure 13a. Suppose that the vertex 8 and its two incident edges $(8,5)$ and $(8,7)$ are removed from $\mathbb{G}$. The resulting graph shown in Figure $13 \mathrm{~b}$ is minimally rigid. The reason is that removing a vertex of degree 2 with its incident edges from a minimally rigid graph is essentially the vertex addition operation in reverse. As explained in $\S 2.4$, this operation preserves minimal rigidity.

The existence of new edges between the neighbors of the removed vertex of degree 3: The vertex 1 is removed with its three incident edges $(1,2),(1,3)$ and $(1,5)$ in $\mathbb{G}$. Then, one of the edges in $\{(2,3),(2,5),(3,5)\}$ is created as shown in Figure $14 \mathrm{a}$ such that the resulting graph is minimally rigid. The edge splitting operation explained in $\S 2.4$ ensures that the addition of at least one edge in $\{(2,3),(2,5),(3,5)\}$ results in a minimally rigid graph.

\section{The existence of new edges between the neighbors of the removed vertex of degree 4 or more:}

For the case of a vertex of degree 3, the edge splitting operation was used to explain the existence of an edge inserted between the neighbors of the removed vertex such that the resulting graph after insertion is minimally rigid. For the cases of removal of vertices of degree 4 and higher, we need to prove the existence of such edges. We have the following lemma for the removal of a vertex of degree 4 [25].

Lemma 19. Let $\mathbb{G}=(\mathcal{V}, \mathcal{E})$ be a minimally rigid graph with a vertex $i$ of degree 4, which has a set of neighboring vertices, $\mathcal{V}_{i}$. Let $\mathbb{G}^{*}$ denote the resulting non-rigid graph after the vertex is removed. Then, for some choice of two non-implicit edges 
among the vertices in $\mathcal{V}_{i}$, the graph $\mathbb{G}^{* *}$ formed from $\mathbb{G}^{*}$ by creating these edges is minimally rigid.

Proof. We choose a minimally rigid graph $\mathbb{G}$ with the vertex $i$ and the vertices in $\mathcal{V}_{i}$. We remove one of the edges of $i$, and call the resulting graph $\mathbb{G}^{\prime}$. To maintain minimal rigidity we create an edge between two points in $\mathcal{V}_{i}$, which is not implicitly present. We refer the reader to Tay and Whiteley [25] for the proof of the existence of such an edge. The proof uses simple linear algebra on the rigidity matrix. If we add this edge, we create $\mathbb{G}^{\prime \prime}$, which is minimally rigid and has a vertex $i$ of degree 3 . We can replace $i$ with a non-implicit edge between two vertices in $\mathcal{V}_{i}$. Hence, a vertex with degree four can be replaced by two edges between the vertices in $\mathcal{V}_{i}$.

Figure 14b shows an example in which the vertex 4 of degree 4 is removed and the edges are created to maintain rigidity.

Now, we have the following lemma to regain rigidity when a vertex of degree 5 or higher is removed from a minimally rigid graph [25].

TheOREM 20. (replacing a vertex of degree $k, k \geq 5$ ) Let $\mathbb{G}=(\mathcal{V}, \mathcal{E})$ be a minimally rigid graph with a vertex $i$ of degree $k$ and with a set of neighboring vertices $\mathcal{V}_{i}$. Then, for some choice of $k-2$ non-implicit edges among the vertices in $\mathcal{V}_{i}$, the graph formed by removing $i$ and the edges whose one end point is $i$, and then adding these $k-2$ edges, is minimally rigid.

Proof. The case for $k=4$ has been shown in Lemma 19. Now we are going to show for $k \geq 5$. Suppose that the theorem holds for $k=n-1$. For $k=n$, we choose a minimally rigid graph $\mathbb{G}$ with vertex $i$ and the set of neighboring vertices $\mathcal{V}_{i}$. We remove one of the edges of $i$, and call the resulting graph $\mathbb{G}^{\prime}$. To maintain minimal rigidity we create an edge between two vertices in $\mathcal{V}_{i}$ which is not implicitly present. We refer the reader to Tay and Whiteley [25] for the proof of the existence of such an edge. If we add this edge, we create $\mathbb{G}^{\prime \prime}$, which is minimally rigid and has a vertex

$i$ with degree $n-1$. From our assumption, we can remove the vertex $i$ by creating $n-3$ new edges. Hence, we can maintain minimal rigidity by creating a total of $n-2$ edges in the case of removing a vertex with degree $n$.

6.2. Spatial Case. Removing a vertex with three edges: Given a minimally rigid graph $\mathbb{G}$ as shown in Figure 15 a, consider the case when the vertex 3 with the three edges $(3,1),(3,2)$ and $(3,5)$ is removed as shown in Figure 15b. For maintaining rigidity, no changes with the remaining vertices and edges need to be made. The reason is that removing a vertex of degree 3 with its incident edges from a minimally rigid graph is essentially the vertex addition operation in reverse.

The existence of new edges between the neighbors of the removed vertex of degree 4: Consider the case in which the vertex 8 with the four edges $(8,2),(8,5)$, $(8,7)$ and $(8,11)$ is removed in $\mathbb{G}$ (Figure 16a). Rigidity is maintained as follows. One of the edges, which is non-implicit in the set $\{(2,5),(2,7),(2,11),(5,7),(5,11),(7,11)\}$ 


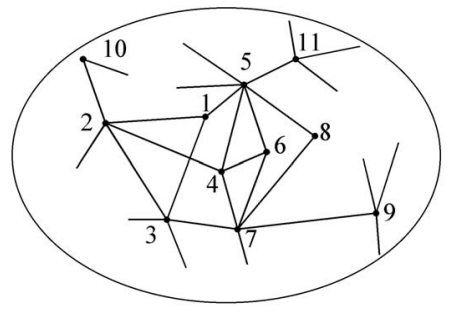

(a)

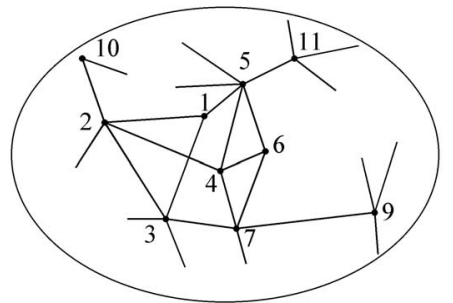

(b)

FIG. 13. (a) The initial graph representing a point formation. (b) The vertex 8 of degree 2 is removed.

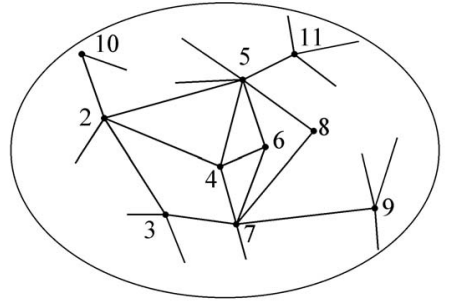

(a)

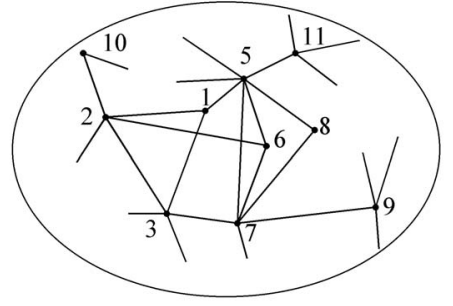

(b)

FIG. 14. (a) The vertex 1 of degree 3 is removed and the edge (2,5) is created to maintain rigidity. (b) The vertex 4 of degree 4 is removed and the edges (2,6),(5,7) are created to maintain rigidity.

(Figure 16b) is created. The edge splitting operation explained in $\S 2.4$ ensures that at least one edge in this set results in a minimally rigid graph.

The existence of new edges between the neighbors of the removed vertex of degree 5 or more: The vertex 7 with five edges is removed as shown in Figure 17a. To maintain rigidity with the remaining vertices, the following theorem is used [25].

Lemma 21. Let $\mathbb{G}=(\mathcal{V}, \mathcal{E})$ be a minimally rigid graph with a vertex $i$ of degree 5 and with a set of neighboring vertices $\mathcal{V}_{i}$. Then, one of the following is true: (i) for some choice of two non-incident edges among the vertices in $\mathcal{V}_{i}$, the graph $\mathbb{G}^{1}$ formed from $\mathbb{G}$ by creating these edges is minimally rigid; or (ii) for two choices of incident pairs of edges among the vertices in $\mathcal{V}_{i}$, (not all incident with a single vertex), the two graphs $\mathbb{G}^{2}$ and $\mathbb{G}^{3}$, formed from $\mathbb{G}$ by creating these pairs of edges, are both minimally rigid. 
In Figure 17b, the edges $(5,10),(6,8)$ are created to maintain rigidity. Now, an agent with six or a higher number of links is removed in a rigid formation. The following theorem is used to maintain rigidity, which has a proof analogous to that of Theorem 20 [25].

TheOREM 22. (replacing a vertex of degree $k, k \geq 6$ ) Let $\mathbb{G}=(\mathcal{V}, \mathcal{E})$ be a minimally rigid graph with a vertex $i$ of degree $k$ and with a set of neighboring vertices, $\mathcal{V}_{i}$. Then, for some choice of $k-3$ non-implicit edges among the vertices in $\mathcal{V}_{i}$, the graph formed by removing $i$ and the edges whose end point is $i$, and adding these $k-3$ edges, is minimally rigid.

It is straightforward to generalize Theorem 18 to cover the case where a minimally rigid graph loses several vertices and their incident edges at the same time.

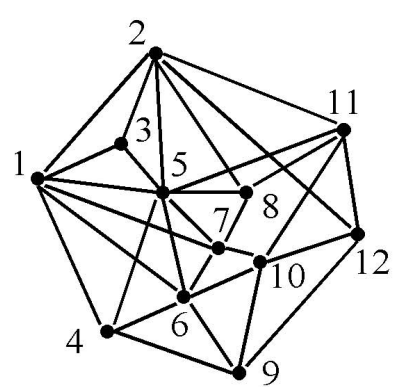

(a)

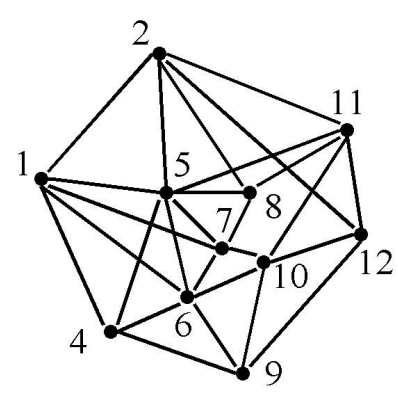

(b)

FIG. 15. (a) A minimally rigid graph in 3-dimensional space. (b) The vertex 3 of degree 3 is removed, and the resulting formation is still rigid.

COROLlaRY 6.1. If two or more vertices are removed from a minimally rigid graph, then the presented techniques in this section can be applied sequentially and new edges can still be inserted just among the neighbors of the removed vertices to ensure rigidity.

Throughout the section, we assumed that the initial graph is minimally rigid. It seems that there will be practical situations where minimality is not present. Such situations may take some effort to analyze but we believe that such cases can be handled with strategies similar to those of this section. We leave their analysis for future work.

7. Summary and Concluding Remarks. In this paper, we have explored topics relevant to rigid formations of autonomous agents. In $\S 2$, we studied the 


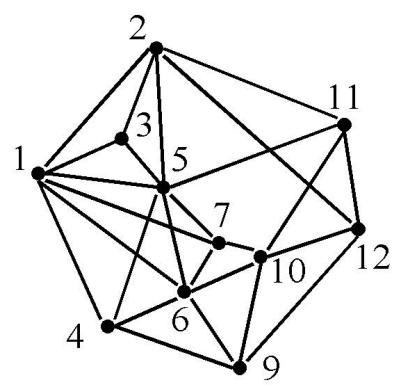

(a)

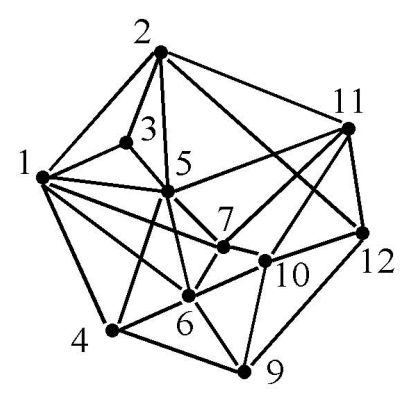

(b)

FIG. 16. (a) The vertex 8 of degree 4 is removed. (b) The link $(7,11)$ is created to maintain rigidity.

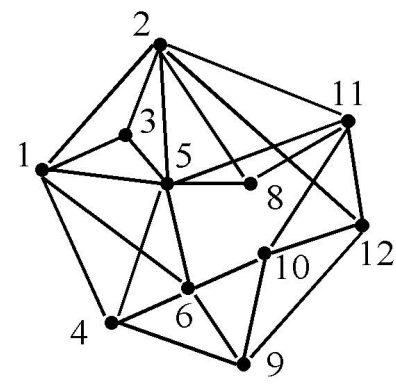

(a)

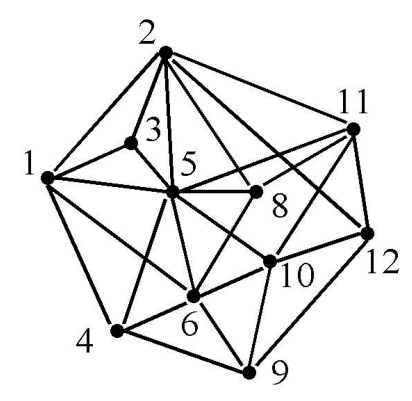

(b)

FIG. 17. (a) The vertex 7 of degree 5 is removed. (b) The links $(5,10),(6,8)$ are created to maintain rigidity.

conditions for rigid formations using distance information between agents and developed techniques to create minimally rigid formations. The framework is scalable for any number of agents. We introduced point formations to model formations of autonomous agents. We reviewed the conditions for rigidity, the rigidity matrix and the combinatorial characterization of rigidity (i.e., Laman's Theorem). Central to the 
development of the approach was rigidity theory, which has been studied in mathematics and structural engineering. $\S 2$ addresses a number of existing problems in the mathematical theory of rigidity. We have been able to translate a number of classical and modern results from rigidity to give a fairly complete theory in 2-dimensional space and partial results in 3-dimensional space. We have also encountered some basic unsolved problems in 3-dimensional space.

In $\S 3$, we studied the minimal cover problem. The splitting and merging problems can be considered as special cases of the minimal cover problem, which is basically to find new links to insert into a non-rigid formation so that it becomes rigid. To solve the minimal cover problem, we develop a novel procedure. In $\S 4$ and $\S 5$, this procedure is used for creating minimally rigid post-split sub-formations from non-rigid post-split sub-formations and for creating a minimally rigid post-merged formation from minimally rigid pre-merged sub-formations, respectively.

In $\S 6$, we studied the closing ranks problem. We developed strategies to regain rigidity when an agent departs from a rigid formation. We considered the following objectives in solving the closing ranks problem: to regain "minimal" rigidity; to preserve remaining sensing and communication links after agent departure; to insert new links only between the neighbors of the departed agent.

To solve the closing ranks problem with local information between agents, the use of triangulations for creating rigid formations is proposed in Eren [9]. With this approach, the new links can be inserted in such a way that local communication between the neighbors of the departed agent is sufficient to determine those new links. Here, we will not go into this approach because of space constraints.

Acknowledgments. Tolga Eren and Peter N. Belhumeur are supported by the National Science Foundation under grants NSF ITR IIS-00-85864, NSF EIA-02-24431 and NSF IIS-03-08185. Brian D. O. Anderson is supported by the Australian Government through the Department of Communications, Information Technologies and the Arts and by the Australian Research Council via a Discovery-project Grant and the Centre of Excellence program. A. Stephen Morse is supported by the National Science Foundation. Walter Whiteley is supported by the Natural Science and Engineering Research Council (Canada) and the National Institutes of Health (USA).

\section{REFERENCES}

[1] B. D. O. Anderson And H. M. Hong, Structural controllability and matrix nets, International Journal on Control, 35(1982), pp. 397-416.

[2] L. Asimow And B. Roth, Rigidity of graphs, Transactions of the American Mathematical Society, 245(1978), pp. 279-289.

[3] L. Berenchtein, L. Chavez, And W. Whiteley, Inductive constructions for 2-rigidity: Basis and circuits via tree coverings, Preprint, York University, Toronto, Ontario, Canada, 2003. 
[4] A. R. Berg And T. Jordan, Algorithms for graph rigidity and scene analysis, In: G. Di Battista and U. Zwick, editors, Lecture Notes in Computer Science, volume 2832, pages 78-89. Springer-Verlag Berlin Heidelberg, 2003.

[5] G. Birkhoff, Lattice Theory. American Mathematical Society, Providence, Rhode Island, 1960.

[6] B. Bollobás, Modern Graph Theory. Springer Verlag, New York, New York, 1998.

[7] J. P. Corfmat And A. S. Morse, Structurally controllable and structurally canonical systems, IEEE Transactions on Automatic Control, February 1976.

[8] H. Crapo And W. Whiteley, Statics of frameworks and motions of panel structures: a projective geometric introduction, Structural Topology, 6(1982), pp. 43-82.

[9] T. EREn, Rigid Formations of Autonomous Agents. Ph.D. thesis, Department of Electrical Engineering, Yale University, New Haven, Connecticut, USA, 2003.

[10] T. Eren, P. N. Belhumeur, B. D. O. Anderson, and A. S. Morse, A framework for maintaining formations based on rigidity, In: Proceedings of the 15th IFAC World Congress, Barcelona, Spain, July 2002.

[11] T. Eren, P.N. Belhumeur, A. S. Morse, B. D. O. Anderson, and W. Whiteley. Splitting and merging formations, Draft preprint, Department of Electrical Engineering, Yale University, New Haven, CT, 2002, March 2002.

[12] H. GLuCK, Almost all simply connected closed surfaces are rigid, In: A. Dold and B. Eckmann, editors, Geometric Topology in Lecture Notes In Mathematics, volume 438, pages 225-239. Springer-Verlag, 1975.

[13] C. Godsil and G. Royle, Algebraic Graph Theory. Springer Verlag, New York, New York, 2001.

[14] J. Graver, A combinatrial approach to infinitesimal rigidity, Preprint, Department of Mathematics, Syracuse University, Syracuse, New York, USA, 1984.

[15] J. Graver, B. Servatius, and H. Servatius, Combinatorial Rigidity. American Mathematical Society, Providence, Rhode Island, 1993.

[16] L. Henneberg, Die Graphische Statik der starren Systeme. Johnson Reprint, 1911.

[17] G. LAman, On graphs and rigidity of plane skeletal structures, Journal of Engineering Mathematics, 4(1970), pp. 331-340.

[18] L. Lovasz And Y. Yemini, On generic rigidity in the plane, SIAM Journal on Algebraic and Discrete Methods, 3(1982), pp. 91-98.

[19] S. Maclane and G. Birkhoff, Algebra. Chealsea Publishing Company, New York, 3rd edition, 1988.

[20] R. Olfati-Saber And R. M. Murray, Graph rigidity and distributed formation stabilization of multi-vehicle systems, In: Proceedings of the 41st IEEE Conference on Decision and Control, Las Vegas, NV, December 2002.

[21] A. Recski, Matroid Theory and its Applications in Electric Network Theory and in Statics. Springer Verlag, 1989.

[22] B. Rотн, Rigid and flexible frameworks, American Mathematical Monthly, 88(1981), pp. 6-21.

[23] B. Roth And W. Whiteley, Tensegrity frameworks, Transactions AMS, 265(1981), pp. 419446.

[24] T. TAY, Linking rigid bodies in n-space. Ph.D. thesis, Department of Mathematics, University of Waterloo, Waterloo, Ontario, Canada, 1982.

[25] T. TAy AND W. Whiteley, Generating isostatic frameworks, Structural Topology, 11(1985), pp. 21-69.

[26] W. Whiteley, Vertex splitting in isostatic frameworks, Structural Topology, 16(1990), pp. 23-30.

[27] W. Whiteley, Matroids from discrete geometry, In: Joseph E. Bonin, James G. Oxley, and Brigitte Servatius, editors, Matroid Theory, volume 197, pages 171-313. American 
Mathematical Society, Contemporary Mathematics, 1996.

[28] W. Whiteley, Rigidity and scene analysis, In: J. Goodman and J. O'Rourke, editors, Handbook of Discrete and Computational Geometry, CRC Press, 2004. 
